\title{
Uncertainty Quantification and Robust Predictive System Analysis for High Temperature Kinetics of $\mathrm{HCN} / \mathrm{O}_{2} /$ Ar Mixture
}

\author{
Sai Hung Cheung, Ernesto Prudencio, and Chris Simmons \\ Institute for Computational Engineering and Sciences, \\ The University of Texas at Austin, 1 University Station Austin, TX, 78712 \\ Kenji Miki* \\ NASA Glenn Research Center, Cleveland, OH, 44135
}

\begin{abstract}
In this paper, a stochastic system based Bayesian approach is applied to quantify the uncertainties involved in the modeling of the $\mathrm{HCN} / \mathrm{O}_{2} / \mathrm{Ar}$ mixture kinetics proposed by Thielen and Roth [1]. This enables more robust predictions of quantities of interest such as rate coefficients of $\mathrm{HCN}+\mathrm{Ar} \rightarrow$ $\mathrm{H}+\mathrm{CN}+\mathrm{Ar}$ and $\mathrm{O}_{2}+\mathrm{CN} \rightarrow \mathrm{NCO}+\mathrm{O}$ by using a stochastic Arrhenius form calibrated against their experimental data. This Bayesian approach requires the evaluation of multidimensional integrals, which cannot be done analytically. Here a recently developed stochastic simulation algorithm, which allows for efficient sampling in the high-dimensional parameter space, is used. We quantify the uncertainties in the modeling of the $\mathrm{HCN} / \mathrm{O}_{2} / \mathrm{Ar}$ mixture kinetics and in turn the two rate coefficients and the other relevant rate coefficients. The uncertainty in the error including both the experimental measurement error and physical modeling error is also quantified. The effect of the number of uncertain parameters on the uncertainties is investigated.
\end{abstract}

Keywords: Bayesian approach, Stochastic system, Uncertainty quantification, Robust predictive analysis, Arrhenius form, Deterministic

\footnotetext{
*Corresponding author

Email addresses: saihung@ices.utexas.edu, prudenci@ices.utexas.edu, and csim@ices.utexas.edu (Sai Hung Cheung, Ernesto Prudencio, and Chris Simmons), kenjiinge@gmail.com (Kenji Miki)
} 
model, Stochastic model, Reaction rate, Experimental error, Modeling error

\section{Introduction}

Because of environmental damage associated with the usage of carbonaceous fuel, measurements of relevant rate coefficients have been an active research subject in the combustion community. One of the most important mechanisms is the formation of pollutant species, e.g., $\mathrm{NO}_{x}$, and in this context, cyano species have been of interest for the purpose of tracking the NO formation and destruction. Kinetics of cyano species are relatively well-understood [1-9], and a very rich database for combustion kinetics is available [10]. However, recent studies reveal that some reactions have a non-Arrhenius type of chemical reaction rate behavior (e.g., $\mathrm{NCO}+\mathrm{O}$ [2], O $+\mathrm{CN}$ [3], $\mathrm{OH}+\mathrm{CH}_{2} \mathrm{O}$ [11], etc.,). This is attributed to the complexity of the reactions such as pressure dependence, the presence of intermediate species, and the existence of other reaction paths. These discoveries may result in a necessity to revisit existing rate coefficient estimates available in the current literature and quantify the uncertainties of these estimates. Moreover, current best practices for the calculation of rate coefficients are also subject to re-examination. Here, we propose a new approach using a stochastic model rather than the commonly used deterministic model explained below.

Due to the complex nature of the hydrocarbon oxidation mechanism, which involves thousands of elementary reactions [12], it is practically impossible to isolate each elementary reaction and calculate the rate coefficient without further simplification. Therefore, one of the conventional ways to determine rate coefficients from observed time-dependent species concentrations is as follows $[1,7,9]$ : First, experimentalists construct a reduced chemistry model based on their knowledge and assumptions, and then calibrate the rate coefficients in order to reasonably reproduce the species concentration profiles. This process is repeated under different experimental conditions (e.g., different temperature) until adequate data are obtained for the purpose of checking the temperature $(T)$ dependence of the rate coefficient $(k)$, which is typically expressed in the Arrhenius form, $k=10^{A} T^{m} \exp \left(-\frac{\Theta}{T}\right)$. Here, the coefficients $(A, m$, and $\Theta$ ) need to be determined. Calibration of these coefficients is not trivial, given the limited amount of experimental data. Also, in this "deterministic-model approach" (i.e., all parameters are single-valued), it is very possible to ignore other possible sets of coefficients. In this sense, 
the standard approach is not robust. Instead, a stochastic system model based on the Bayesian approach presented in the next section seems to be a powerful alternative, reflecting the current state of knowledge, including uncertainty.

There are several notable studies related to the uncertainty quantification involving chemical reaction systems, e.g., [13-17]. These papers mostly focused on the statistical forward problem including sensitivity analysis in which the uncertainties in chemical quantities of interest were quantified by propagating some prescribed uncertainties in the model parameters (such as those in the rate coefficients) through the chemical model equations. There are other papers (e.g., [18-27]) focusing on the inverse problem in which the model parameters (those that are used to parametrize the rate coefficients) were determined by minimizing some error functions considering experimental data or data obtained by the detailed mechanics. For instance, in $[18,19]$, probabilistic dependence among the unknown model parameters cannot be easily quantified, and the form of probability density function needs to be prescribed a priori. (As explained later, we also assume a Gaussian distribution for the experimental measurements based on the Central Limit Theorem, but not for the model parameters) Moreover, the parameters of the probability density function have to be assumed fixed at the solution of a minimization problem. This leads to "underestimation" in the sense that there are significant uncertainties due to the assumed form of probability density function and also the unknown parameters for such probability density function. Recently Nagy and Turányi [28] investigated the uncertainties associated with the expression of the rate coefficient in the Arrhenius form and emphasized the importance of considering the correlation among the Arrhenius coefficients in the varying temperature chemical kinetic systems. Similarly, Prager et al. [27] performed the inverse problem using Polynomial chaos expansions (PCEs) coupled with Bayesian inference model and calibrated model parameters including Arrhenius parameters of propane/methane/air mixture in an ignition delay problem. They highlighted the important of taking into account the correlations among these parameters (i.e., joint probability density function), which is consistent with our previous studies $[25,26]$

In this work, our focus is on a more complete and flexible quantification of the uncertainty in modeling the chemical reaction systems. The approach presented in this paper allows the prior probability density function of any unknown parameters to be updated using the measured data to the posterior probability density function while simultaneously quantifying the uncertainty 
of the errors used to quantify the model inadequacy. All the probability information including any probabilistic dependence among the uncertain model parameters or any statistical quantities such as higher moments is automatically encapsulated in the posterior probability density function that can be propagated forward to obtain the uncertainty in the chemical quantities of interest relevant to the model whose uncertainty is being quantified. Problems with many uncertain model parameters and a very complicated probability density function can be solved with the help of a state of the art stochastic simulation algorithm such as the one adopted in this paper.

Moreover, we revisit the study of $\mathrm{HCN} / \mathrm{O}_{2} / \mathrm{Ar}$ mixture kinetics done by Thielen and Roth [1]. In their paper, two reactions related to the cyano species formation/destruction were investigated:

$$
\begin{array}{r}
\mathrm{HCN}+\mathrm{Ar} \rightarrow \mathrm{H}+\mathrm{CN}+\mathrm{Ar} \quad(R 1), \\
\mathrm{O}_{2}+\mathrm{CN} \rightarrow \mathrm{NCO}+\mathrm{O} \quad(R 3) .
\end{array}
$$

Here, $R 1$ is the thermal decomposition of $\mathrm{HCN}$, and the model coefficients in the Arrhenius form for a certain temperature range have been obtained by several researchers [29-31]. It can be seen that the model coefficients obtained by these researchers show large variation (See Table 1 which includes the result from [1]). For example, the pre-exponential term $10^{A}$ varies from $4 \times 10^{14} \mathrm{~cm}^{3} \mathrm{~mol}^{-1} \mathrm{~s}^{-1}$ to $3 \times 10^{22} \mathrm{~cm}^{3} \mathrm{~mol}^{-1} \mathrm{~s}^{-1}$ depending on the value of $m$ and the characteristic temperature associated with the activation energy, $\Theta$. However, all of them follow the trend for a certain temperature range except those of Tabayashi who used a different absorption coefficient for CN. This large variation of coefficients is related to the nature of nonlinear temperature dependence of the rate coefficient in the Arrhenius form, and thus a sufficiently large amount of data is required over a broad range of temperature to obtain a consistent value. Moreover, as mentioned above, uncertainty of other reactions used in the simplified mechanism can bring in additional uncertainties in the rate of $R 1$ due to the interactions of these reactions (e.g., Thielen and Roth considered the reaction: $\mathrm{HCN}+\mathrm{O} \rightarrow \mathrm{NCO}+\mathrm{H}(R 4)$ that has two orders of magnitude range of variation [32]).

There are several publications studying the rate coefficient for $R 3$ (e.g., Mulvihill and Phillips [8], Thielen and Roth [1] and Baulch et al. [33]). Their results for the rate coefficient differ by about one order of magnitude at $T=2500 \mathrm{~K}$ with the former showing temperature dependence and the latter being temperature invariant $\left(k_{3}=4.90 \times 10^{12} \mathrm{~cm}^{3} \mathrm{~mol}^{-1} \mathrm{~s}^{-1}\right)$. The explanation for this discrepancy given by Thielen and Roth was that the variance 
of experimental data obtained by Mulvihill and Phillips [8] was too large. On the other hand, the thermal decomposition of NCO considered in the simplified model for the determination of the rate coefficient of $R 3$ in [1] has large uncertainties through the other rate coefficients associated with the oxidation of $\mathrm{NCO}$ (e.g., $\mathrm{NCO}+\mathrm{O} \rightarrow \mathrm{CO}+\mathrm{NO}(R 18)[34])$.

The objective of this study is to investigate the rate coefficients of $R 1$ and $R 3$ and to quantify their corresponding uncertainties based on the experimental data presented in [1] using the stochastic system based Bayesian approach. For the purpose of comparing the difference between the results from the "deterministic-model" approach [1] and the proposed stochastic system model based Bayesian approach, we consider the same simplified set of reactions (the 6 reactions: $R 1-R 6$ ) and experimental data as presented in [1].

The layout of the paper is as follows. In Sec. 2, we present the simplified chemical system considered in [1] and then construct stochastic models for the uncertainty quantification of the chemical reaction system. In Sec. 4, a detailed comparison between our results with previous work is provided, and the robustness of the current approach is discussed. The conclusions are summarized in Sec. 5.

\section{Numerical studies}

\subsection{Kinetics of $\mathrm{HCN} / \mathrm{O}_{2} / \mathrm{Ar}$ mixture and experimental data}

Here, we consider $\mathrm{HCN} / \mathrm{O}_{2}$ chemistry with 11 species $\left(\mathrm{O}, \mathrm{N}, \mathrm{H}, \mathrm{Ar}, \mathrm{O}_{2}\right.$, $\mathrm{OH}, \mathrm{CN}, \mathrm{CO}, \mathrm{NO}, \mathrm{HCN}$, and $\mathrm{NCO}$ ) and 6 reactions [1]:

$$
\begin{array}{rc}
\mathrm{HCN}+\mathrm{Ar} \leftrightarrow \mathrm{H}+\mathrm{CN}+\mathrm{Ar} & (R 1), \\
\mathrm{O}_{2}+\mathrm{H} \leftrightarrow \mathrm{OH}+\mathrm{O} & (R 2), \\
\mathrm{O}_{2}+\mathrm{CN} \leftrightarrow \mathrm{NCO}+\mathrm{O} & (R 3), \\
\mathrm{HCN}+\mathrm{O} \leftrightarrow \mathrm{NCO}+\mathrm{H} & (R 4), \\
\mathrm{NCO}+\mathrm{Ar} \leftrightarrow \mathrm{CO}+\mathrm{N}+\mathrm{Ar} & (R 5), \\
\mathrm{O}_{2}+\mathrm{N} \leftrightarrow \mathrm{NO}+\mathrm{O} \quad(R 6) .
\end{array}
$$

A detailed explanation of the choice of this reduced mechanism is given by Thielen and Roth [1]. To compare the results obtained by the "deterministicmodel" approach and the stochastic system model based Bayesian approach, we start with the above reduced set of chemical reactions and consider the same data set that Thielen and Roth [1] used to predict the rate coefficient for 
$R 3$. For the rate coefficient of $R 1$, they used previous experimental data [35] and they fixed the values of the Arrhenius parameters of $R 1$ when calibrating $R 3$. Using this model, they reproduced the time-histories of concentration of $\mathrm{O}, \mathrm{N}$, and $\mathrm{H}$ at early stages $(\leq 800 \mu s)$ when the concentration of other radical species (e.g., $\mathrm{NH}, \mathrm{HCN}, \mathrm{NCN}$, etc.,) and products (e.g., $\mathrm{H}_{2} \mathrm{O}, \mathrm{N}_{2}$, and $\mathrm{CO}_{2}$ ) was relatively low (see Fig. 1). A brief physical explanation of these six reactions is given as follows: $R 1$ is a critical rate limiting reaction that significantly influences the initial profiles of all species concentrations by producing radicals, $\mathrm{H}$ and $\mathrm{CN}$ as a result of thermal decomposition of $\mathrm{HCN}$. These radicals facilitate the dissociation reaction with $\mathrm{O}_{2}$ whose dissociation energy is relatively low. As the population of $\mathrm{O}$ increases, the additional pathway of decomposition of $\mathrm{HCN}$ with $\mathrm{O}$ needs to be included $R 4$. In their model, they did not include $\mathrm{HCN}+\mathrm{O} \rightarrow \mathrm{NH}+\mathrm{CO}(R 9)$ and $\mathrm{HCN}+$ $\mathrm{O} \rightarrow \mathrm{CN}+\mathrm{OH}(R 10)$ (Note that the complexity of the reaction between $\mathrm{HCN}$ and $\mathrm{O}$ is related to the fact that the intermediate species $\mathrm{HCNO}$ is formed). However, Roth et al. [32] used the same rate coefficient of $R 4$ to represent the combination of $R 4$ and $R 9$ with large uncertainties. Another value for the rate coefficient of $R 4$ was obtained by Louge and Hanson [34]. They reported $k_{4, \text { Louge }}=10^{8.24} T^{1.47} \exp (-3775 / T)$ that is substantially different from the form used in Thielen and Roth $\left(k_{4}=10^{13.86} \exp (-7460 / T)\right)$. Perry and Melius suggested the rate coefficient of $\mathrm{HCN}+\mathrm{O} \rightarrow$ product is $10^{12.77} \exp (-4030 / T)$ [36]. We will discuss the dependence between the uncertainties in the predicted rate coefficients of $R 1$ and $R 3$ and the uncertainty in $R 4$ later. NCO is generated by the major pathway between $\mathrm{HCN}$ and $\mathrm{NCO}$, i.e., $R 4$, and is dissociated by Ar into $\mathrm{N}$ and $\mathrm{CO}$. This is an important reaction to produce $\mathrm{N}$ atoms as a counterpart to $R 6$ where $\mathrm{N}$ brings in chain-carrying reactions.

The concentrations of $\mathrm{N}, \mathrm{O}$, and $\mathrm{H}$ were measured for the temperature range $2195 \mathrm{~K} \leq \mathrm{T} \leq 3390 \mathrm{~K}$ in the pressure range $1.5-1.9$ bar [1]. The initial concentration of $\mathrm{HCN}$ was varied between 25 parts per million (ppm) and $200 \mathrm{ppm}$, and that of $\mathrm{O}_{2}$ was varied between $50 \mathrm{ppm}$ and $1000 \mathrm{ppm}$. The concentrations of each atom were measured with different background gas pressure ratios, $[\mathrm{HCN}] /\left[\mathrm{O}_{2}\right]$, at six different times $(100 \mu s, 200 \mu s, 300 \mu s$, $400 \mu s, 600 \mu s$, and $800 \mu s$ ). The experimental uncertainty for concentration measurement was reported to be: $\pm 25 \%$ for $\mathrm{N}$ and $\pm 15 \%$ for $\mathrm{O}$ and $\mathrm{H}$. This information can be used as a reference to construct the prior for the stochastic model class presented later taking into account the fact that the experimental error reported in the literature may not be accurate. Following the argument 
given by Thielen and Roth [1], we use only the data corresponding to the experiments with $[\mathrm{HCN}] /\left[\mathrm{O}_{2}\right]=1$ for this study.

\subsection{Formulation of Deterministic model}

Assuming that the temperature is constant during the reaction due to the mixture being highly diluted by Ar, the chemical reactions can be expressed in a simplified form by introducing the stoichiometric coefficients $\left(\nu_{m, r}^{\prime}\right.$ and $\nu_{m, r}^{\prime \prime}$ for the reactant and product of the $r$-th reaction):

$$
\sum_{m=1}^{N_{s}} \nu_{m, r}^{\prime} X_{m} \rightleftharpoons \sum_{m=1}^{N_{s}} \nu_{m, r}^{\prime \prime} X_{m}
$$

where $N_{s}$ and $X_{m}$ are the number of species in the chemical reaction set (11 for this study) and the mole fraction of the $m$-th species. Thus, the chemical reaction of $X_{m}$ in the $r$-th reaction is given by:

$$
\begin{array}{r}
\frac{d\left[X_{m}\right]}{d t}=\sum_{r=1}^{N_{r}}\left\{\left(\nu_{m, r}^{\prime \prime}-\nu_{m, r}^{\prime}\right) k_{f, r} \prod_{m=1}^{N_{s}}\left[X_{m}\right]^{\nu_{m, r}^{\prime}}+\right. \\
\left.\left(\nu_{m, r}^{\prime}-\nu_{m, r}^{\prime \prime}\right) k_{b, r} \prod_{m=1}^{N_{s}}\left[X_{m}\right]^{\nu_{m, r}^{\prime \prime}}\right\}
\end{array}
$$

The forward rate coefficient of the $r$-th reaction, $k_{f, r}$, is calculated using the modified Arrhenius equation: $k_{f, r}=10^{A_{r}} T^{m_{r}} \exp \left(-\frac{\Theta_{r}}{T}\right) \quad r=1, \ldots, N_{r}=6$, where $A_{r}$ and $m_{r}$ are the pre-exponential Arrhenius parameters and $\Theta_{r}$ is the characteristic temperature of the activation energy. The corresponding backward rate coefficient, $k_{b, r}$, is obtained by $k_{b, r}=k_{f, r} / K_{C, r}$ where $K_{C, r}$ is the equilibrium constant.

\section{Stochastic system model based Bayesian approach}

\subsection{Bayesian approach}

A stochastic system model based framework for Bayesian model updating presented in $[37,38]$ was adopted in this paper. A key concept here is a stochastic system model class $M$ with uncertain model parameters $\boldsymbol{\theta} \in \Omega \subset$ $\mathbb{R}^{d}$, which consists of 1 ) a chosen set of probabilistic or stochastic inputoutput predictive models for a system; 2) a chosen probability distribution, the prior, over this set that quantifies the initial relative plausibility of each predictive model in the set.

Based on a stochastic system model class $M$, one can use data $D$ to compute the updated relative plausibility of each predictive model in the 
set defined by $M$. This is quantified by the posterior probability density function (PDF) $p(\boldsymbol{\theta} \mid D, M)$ for the uncertain model parameters $\boldsymbol{\theta} \in \Omega \subset \mathbb{R}^{d}$, which specify a particular stochastic model within $M$. By Bayes' theorem, this posterior PDF is given by:

$$
p(\boldsymbol{\theta} \mid D, M)=c^{-1} p(D \mid \boldsymbol{\theta}, M) p(\boldsymbol{\theta} \mid M)
$$

where $c$ is the normalizing constant which makes the probability volume under the posterior PDF equal to unity; $p(D \mid \boldsymbol{\theta}, M)$ is the likelihood function which expresses the probability of getting data $D$ (measurement of some system outputs) based on the predictive PDF for the system output given by model $\boldsymbol{\theta}$ within $M$; and $p(\boldsymbol{\theta} \mid M)$ is the prior PDF for $\boldsymbol{\theta}$ which one uses to quantify the initial plausibility of each model defined by the value of the parameters $\boldsymbol{\theta}$ based on expert knowledge or past knowledge obtained from data. As emphasized by Jaynes [39], probability models represent a quantification of the state of knowledge about real phenomena conditional on the available information and should not be imagined as a property inherent in these phenomena.

The likelihood function, which can be obtained using the Theorem of Total Probability $[37,40]$, expresses the probability of getting $D$ given the predictive model $\boldsymbol{\theta}$ within $M$ :

$$
p(D \mid \boldsymbol{\theta}, M)=\int p(D \mid \mathbf{v}, \boldsymbol{\theta}, M) p(\mathbf{v} \mid \boldsymbol{\theta}, M) d \mathbf{v},
$$

where $\mathbf{v}$ is comprised of all the unobserved model outputs, model states, modeling and measurement errors, boundary conditions, and initial conditions, etc.

\subsection{A probabilistic input-output predictive model for the chemical reaction system}

It will be seen later that the stochastic models formed by embedding probability models into the system of ODEs for the chemical reactions are actually a special case of input-output models given as follows ([37, 40-42]):

$$
\begin{aligned}
& \mathbf{w}(\mathbf{x}, \boldsymbol{\theta}, \boldsymbol{\gamma}, \mathbf{u})=\mathbf{0} \\
& \mathbf{y}_{\text {model }}=\mathbf{y}_{\text {model }}(\mathbf{x}, \boldsymbol{\theta}, \boldsymbol{\varepsilon}, \mathbf{u}) \\
& \mathbf{y}=\mathbf{y}\left(\boldsymbol{\theta}, \mathbf{y}_{\text {true }}, \boldsymbol{\xi}, \mathbf{u}\right)
\end{aligned}
$$

where the state $\mathbf{x}$ is the solution of the stochastic equation $\mathbf{w}(\mathbf{x}, \boldsymbol{\theta}, \boldsymbol{\gamma}, \mathbf{u})=\mathbf{0}$ which is constructed by embedding to the base model $\gamma$ which can be modeled 
as random vectors or random fields and $\mathbf{u}$ can denote scenarios or any other inputs; $\mathbf{y}_{\text {model }}$ is the stochastic model output with $\varepsilon$ as random vectors or random fields; $\mathbf{y}_{\text {true }}$, which is some subset of $\mathbf{y}_{\text {model }}$, is the actual quantity corresponding to $\mathbf{y}$ which is to be measured and is modeled as a random vector or random field and $\boldsymbol{\xi}$ which are random vectors or random fields, that can be used to model the measurement noise and the measured/observed data $D$ is a realization of $\mathbf{y} ; \boldsymbol{\gamma}, \boldsymbol{\varepsilon}$ and $\boldsymbol{\xi}$ are random vectors or random fields with some joint PDF $p\left(\boldsymbol{\gamma}, \boldsymbol{\varepsilon}, \boldsymbol{\xi} \mid \boldsymbol{\theta}_{\boldsymbol{e}}, M_{j}\right)$ where $\boldsymbol{\theta}_{\boldsymbol{e}}$ consists of some subset of the uncertain parameter vector $\boldsymbol{\theta}$ of $M$. The type of embedded stochastic models (for $\boldsymbol{\gamma}, \boldsymbol{\varepsilon}$ and $\boldsymbol{\xi}$ as above) can be chosen based on Jaynes' Principle of Maximum Information Entropy [39].

So for the problem of interest in this paper, $\mathbf{x}$ is the time histories of concentration of all species involved; the base model is given by the stiff ODEs; $\mathbf{y}_{\text {model }}$ gives the time histories of concentration of some species predicted by the model; the uncertain parameters include the parameters of the base model, for example, Arrhenius parameters which are used to parametrize the rate coefficients as a function of temperature and the parameters of the embedded stochastic models. Thus, the stochastic models for this study take the following form for the $i$-th experiment, $i=1,2, \ldots, N_{\text {exp }}\left(N_{\text {exp }}\right.$ is the number of experimental data sets and is set to 22) [25, 26]:

$$
\begin{aligned}
& \mathbf{x}_{0}=\mathbf{x}_{0}\left(\mathbf{u}_{0}^{(i)}\right) \\
& \mathbf{x}_{n}=\mathbf{f}_{n-1}\left(\mathbf{x}_{n-1}, \mathbf{u}_{n-1}^{(i)} ; \boldsymbol{\theta}\right)+\gamma_{n-1}^{(i)} \in \mathbb{R}^{N_{s}=11} \\
& y_{n}^{(i)}=x_{n, w} \exp \left(\varepsilon_{n}^{(i)}\right) \in \mathbb{R}, n=1, \ldots, N_{T}=6
\end{aligned}
$$

where $\mathbf{u}_{n}^{(i)}$ is the vector storing the temperature, pressure, and $\mathrm{HCN} / \mathrm{O}_{2} / \mathrm{Ar}$ concentrations in the $i$-th experiment at time $t_{n}$, and the $m$-th component of $\mathbf{x}_{n}, x_{n, m}$ is the concentration of the $m$-th species at time $t_{n}$ where $n=$ $1, \ldots, N_{T}\left(N_{T}\right.$ is the number of time instance of measuring the concentration at each experiment). The set of measured data $(D)$ is compared with the predictions, $y_{n}^{(i)}$. In each experiment, the species to be measured is either oxygen $(w=1)$ or nitrogen $(w=2)$. The $m$-th component of $\mathbf{f}_{n-1}$ is given by the solution (i.e., concentration of the $m$-th species) at time $t_{n}$. It should be noted that the form of the observation output equation in Eq. (6) is chosen so that it agrees with the non-negativeness of the data $[25,26]$. The term $\exp \left(\varepsilon_{n}^{(i)}\right)$ can be viewed as a type of multiplicative error which accounts for different types of errors including modeling error and experimental error. 
The probability models of the dynamic model equation error $p\left(\left[\gamma_{1}^{(i) T}, \ldots\right.\right.$, $\left.\left.\gamma_{N_{T}}^{(i) T}\right]^{T} \mid \boldsymbol{\theta}, M\right)$ and the output equation error $p\left(\left[\varepsilon_{1}^{(i)}, \ldots, \varepsilon_{N_{T}}^{(i)}\right]^{T} \mid \boldsymbol{\theta}, M\right)$ are chosen to be Gaussian (Justified by the Principle of Maximum Information Entropy). The use of such a distribution is very common in Bayesian analysis due to its flexibility to capture the uncertain discrepancy between what can be predicted by the underlying physical model and the experimental data. The mean of these Gaussian PDFs is chosen to be zero and the corresponding covariance matrices are treated as uncertain. For illustration in this study, the covariance matrix for $p\left(\left[\gamma_{1}^{(i) T}, \ldots, \gamma_{N_{T}}^{(i) T}\right]^{T} \mid \boldsymbol{\theta}, M\right)$ is chosen to be zero, and $p\left(\left[\varepsilon_{1}^{(i)}, \ldots, \varepsilon_{N_{T}}^{(i)}\right]^{T} \mid \boldsymbol{\theta}, M\right)$ is chosen to be an independently distributed Gaussian with each component having a zero mean and a variance equal to $\sigma_{\text {total }}^{2}$ which is the last component of the uncertain parameter vector $\boldsymbol{\theta}$ :

$$
p\left(\left[\varepsilon_{1}^{(i)}, \ldots, \varepsilon_{N_{T}}^{(i)}\right]^{T} \mid \boldsymbol{\theta}, M\right)=\frac{1}{\left\{2 \pi \sigma_{\text {total }}^{2}\right\}^{\frac{N_{T}}{2}}} \exp \left\{-\frac{1}{2} \sum_{n=1}^{N_{T}} \frac{\varepsilon_{n}^{(i) 2}}{\sigma_{\text {total }}^{2}}\right\}
$$

More complicated probability models (such as one with more complicated covariance functions for the noise and error terms [43]) and different error and noise structures will be considered in a future paper. For the above, $\sigma_{\text {total }}^{2}$ can further be written as the sum of two variances $\sigma_{\text {exp }}^{2}$, the variance for the log multiplicative measurement (experimental) error $\varepsilon e_{n}^{(i)}$ and $\sigma_{p}^{2}$, the variance of all other multiplicative errors $\varepsilon p_{n}^{(i)}$ (including output modeling error corresponding to the physical modeling, which is simply called output modeling error in this paper) corresponding to the system quantities that are measured in the experiments. For this model, the output prediction error is then the product of these two types of errors appearing in the output measurement equation, the $\log$ of which has variance $\sigma_{\text {exp }}^{2}$ and $\sigma_{p}^{2}$, respectively. Mathematically, it is equivalent to replace the product of these errors at each time $n$ in the $i$-th experiment by a single multiplicative error term as in Eq. (6) whose $\log$ has variance equal to $\sigma_{\text {total }}^{2}=\sigma_{p}^{2}+\sigma_{\text {exp }}^{2}$. These two log error variances are indistinguishable without prior information since the data only provides information for their sum. If an accurate knowledge of experimental uncertainty is available, the PDF for $\sigma_{\text {exp }}^{2}$ can be constructed taking this into account. The posterior PDF for $\sigma_{p}^{2}$ can then be determined based on the posterior PDF for $\sigma_{\text {total }}^{2}$, and the PDF for $\sigma_{\text {exp }}^{2}$. The prior PDF for $\sigma_{\text {total }}^{2}$ is chosen to be a uniform distribution over the interval $\left[0,(\log (0.3) / 2)^{2}\right]$ so that the prior PDF for the total multiplicative error $r_{\text {total, } n}^{(i)}=\exp \left(\varepsilon_{n}^{(i)}\right)$ is 
approximately a log-normal distribution with median equal to 1 , and most of the probability is within the range $[-90 \%,+300 \%]$ from 1 (See Fig. 2).

For the stochastic system model class $M$ considered in this study, we further assume $p\left(\left[\varepsilon_{1}^{(i)}, \ldots, \varepsilon_{N_{T}}^{(i)}\right]^{T} \mid \boldsymbol{\theta}, M\right)$ for different $i$ (that is for different experiments) are independently and identically distributed:

$$
\begin{aligned}
& p\left(\left[\varepsilon_{1}^{(1)}, \ldots, \varepsilon_{N_{T}}^{(1)}, \ldots, \varepsilon_{1}^{\left(N_{e x p}\right)}, \ldots, \varepsilon_{N_{T}}^{\left(N_{e x p}\right)}\right]^{T} \mid \boldsymbol{\theta}, M\right) \\
& =\frac{1}{\left\{2 \pi \sigma_{\text {total }}^{2}\right\}^{\frac{\sum_{i=1}^{N_{\text {exp }} N_{T}}}{2}}} \exp \left\{-\frac{1}{2} \sum_{i=1}^{N_{\text {exp }}} \sum_{n=1}^{N_{T}} \frac{\varepsilon_{n}^{(i) 2}}{\sigma_{\text {total }}^{2}}\right\}
\end{aligned}
$$

The likelihood function $p(D \mid \boldsymbol{\theta}, M)$ can be derived using Eq. (4) with $\mathbf{v}$ being $\left[\varepsilon_{1}^{(1)}, \ldots, \varepsilon_{N_{T}}^{(1)}, \ldots, \varepsilon_{1}^{\left(N_{e x p}\right)}, \ldots, \varepsilon_{N_{T}}^{\left(N_{e x p}\right)}\right]^{T}$ and $p(\mathbf{v} \mid \boldsymbol{\theta}, M)$ given by Eq. (8) and according to Eq. (6), it can be seen that $p(D \mid \mathbf{v}, \boldsymbol{\theta}, M)$ is given by the following with $D$ being a realization of $\mathbf{y}=\left[y_{1}^{(1)}, \ldots, y_{N_{T, 1}}^{(1)}, \ldots, y_{1}^{\left(N_{e x p}\right)}, \ldots, y_{N_{T}}^{\left(N_{e x p}\right)}\right]^{T}$ (i.e., substituting $\mathbf{y}$ by $D$ ):

$$
p(\mathbf{y} \mid \mathbf{v}, \boldsymbol{\theta}, M)=\delta(\mathbf{y}-\mathbf{g}(\boldsymbol{\theta}, \mathbf{v}))
$$

where $\mathbf{g}(\boldsymbol{\theta}, \mathbf{v})$ is given by:

$$
\mathbf{g}(\boldsymbol{\theta}, \mathbf{v})=\left[\begin{array}{c}
x_{1, w_{1}}(\boldsymbol{\theta}) \varepsilon_{1}^{(1)} \\
\vdots \\
x_{N_{T}, w_{1}}(\boldsymbol{\theta}) \varepsilon_{N_{T}}^{(1)} \\
\vdots \\
x_{1, w_{N_{e x p}}}(\boldsymbol{\theta}) \varepsilon_{1}^{\left(N_{e x p}\right)} \\
\vdots \\
x_{N_{T}, w_{N_{e x p}}}(\boldsymbol{\theta}) \varepsilon_{N_{T}}^{\left(N_{e x p}\right)}
\end{array}\right]
$$

Thus, it can be seen that for a given $\boldsymbol{\theta}, y_{n}^{(i)}$ follows a lognormal distribution with median equal to $x_{n, w}(\boldsymbol{\theta})$ (there is an equal probability for $y_{n}^{(i)}$ going above and below this value) and $\log$ standard deviation $\sigma_{\text {total }}$. From the above, It can be shown that $\mathbf{y}$ is a product of lognormal distributions, and the likelihood function $p(D \mid \boldsymbol{\theta}, M)$ is then given as follows [25, 26]:

$$
\begin{aligned}
& p(D \mid \boldsymbol{\theta}, M)=\frac{1}{\left(2 \pi \sigma_{\text {total }}^{2}\right)^{\frac{\sum_{i=1}^{N_{\text {exp }}} N_{T}}{2}} \prod_{i=1}^{N_{\text {exp }}} \prod_{n=1}^{N_{T}} y_{n}^{(i)}} \\
& \exp \left\{-\frac{1}{2} \sum_{i=1}^{N_{\text {exp }}} \sum_{n=1}^{N_{T}} \frac{\left[\ln \left(y_{n}^{(i)}\right)-\ln \left(x_{n, w}\right)\right]^{2}}{\sigma_{\text {total }}^{2}}\right\}
\end{aligned}
$$


Note that the proper choice of the likelihood function is very important and has a critical influence on the accuracy of the error estimation. Therefore, the results shown later are conditioned by Eq. (11). In fact, different probabilistic models can be used depending on the error structure (either additive or multiplicative) and the different descriptions of the statistical correlation among data points. Selecting the most suitable model for a given set of data is not a trivial task. One possible way to evaluate the performance of each probabilistic model is to consider the deviance information criterion (DIC) $[44,45]$. Such a model comparison is outside the scope of this paper.

\subsection{Formulation of the candidate stochastic system model class $M$}

Let the values given in [1] (as shown in Table 2) be the nominal values of the Arrhenius parameters of the six reactions and be used to normalize the parameters (e.g., $\tilde{A}_{r}, \tilde{m}_{r}$, and $\tilde{\Theta}_{r}, r=1, \ldots, 6$ ). Let the nominal value of $\sigma_{\text {total }}^{2}$ be $(\log (0.3) / 2)^{2}$. In the following section, we will investigate the effect of the number of uncertain parameters on the uncertainties in the rate coefficients and the prediction of the time evolution of species concentrations. Dependence on different model parameters and different rate coefficients is

also investigated. At first, besides $\sigma_{\text {total }}^{2}$, we will treat model parameters of $R 1\left(A_{1}, m_{1}\right.$, and $\left.\Theta_{1}\right)$ as uncertain while those corresponding to the other reactions are fixed at the nominal values. We will refer to this as case 1 and the corresponding stochastic model class $M_{1}$ with four uncertain parameters. For case 2 (denoting the corresponding stochastic model class with seven uncertain parameters $M_{2}$ ), we relax this assumption and consider the model parameters of $R 3$ as additional uncertain parameters. Next for case 3 (denoting the corresponding stochastic model class with ten uncertain parameters $M_{3}$ ), we consider all model parameters of $R 4$ as additional uncertain parameters. Finally for case 4 (denoting the corresponding stochastic model class with nineteen uncertain parameters denoted by $M_{4}$ ), we consider all model parameters of all reactions to be uncertain. The number of the model parameters increases from $4\left(M_{1}\right)$ to $19\left(M_{4}\right)$. Obviously, $\boldsymbol{\theta}$ depends on which model class is being considered. For convenience, the subscript of $\boldsymbol{\theta}$ which is used to refer to which model class $\boldsymbol{\theta}$ belongs is left implicit. The prior PDF for $\boldsymbol{\theta}$ is chosen to be the product of the prior PDFs for each uncertain parameter in $\boldsymbol{\theta}$ (assuming independence among uncertain parameters). Taking the information given in Table 1 and past studies for relevant reactions [46] into account, the prior PDFs for $A_{i}, m_{i}$, and $\Theta_{i}$ are chosen as uniform distributions on the interval shown in Table 3 . Large intervals are assigned to 
the prior of $A_{i}$ due to the choice of the values of $m$. Also, for $\Theta_{3}$, the distribution covers the domain with negative values in order to cover all reported values [46].

It is important to notice that a large interval of the prior enables us to calibrate the random variables with the possibility of evaluating their stochastic dependency (e.g., higher moment). This is very important especially when we aim at understanding the temperature dependence of uncertainties in rate coefficients. This problem is recently discussed by Nagy and Turányi [28] and Prager et al. [27] In fact, it is much easier to calibrate only the A term covering all previously reported rate constants. Indeed, this type of calibration has been often performed before, but this implicitly assumes that the uncertainty in the rate coefficient is independent of temperature. This is not the case. Because of complex correlations among the terms: A, m, and $\Theta$, it is not easy to set a "reasonable" interval of the prior. We choose large uniform priors of Arrhenius parameters so that the ranges are large enough not to influence the posterior distributions [27]. Also, from the computational viewpoint, it is not favorable to use a too large interval for the prior, which leads to slow convergence. To compromise these issues, our choice of the prior should be acceptable.

\subsection{Summary of the procedures for quantifying uncertainties}

A brief summary of the main procedures for quantifying uncertainties using the above Stochastic system model based Bayesian approach is described as follows:

1. Construct each stochastic model class by choosing a set of a chosen set of probabilistic or stochastic input-output predictive models for a system and a chosen probability distribution, the prior, over this set that quantifies the initial relative plausibility of each predictive model in the set. Probabilistic input-output predictive models can be constructed using the base model (for example, the chemical model in this study) as the starting point and embedding stochastic models to it (following the procedures given above) to be in the form given by, for example, Eq. (5).

2. From Eq. (5) and all other assumptions in $M$, one can obtain the likelihood function, Eg. (11)

3. The uncertainty information of the uncertain model parameters $\boldsymbol{\theta}$ quantified by the data is encapsulated in the posterior $\operatorname{PDF} p(\boldsymbol{\theta} \mid D, M)$ for $\boldsymbol{\theta}$ 
given by Eq. (3) which can be approximated by:

$$
p(\boldsymbol{\theta} \mid D, M) \approx \frac{1}{K} \sum_{k=1}^{K} \delta\left(\boldsymbol{\theta}-\boldsymbol{\theta}^{(k)}\right)
$$

where the samples ( $K$ is the sample size) can be obtained by stochastic simulation procedures such as [40].

4. Robust predictions (including uncertainty quantification) of any quantities of interest $(\mathbf{Q}), p(\mathbf{Q} \mid D, M)$, can be approximated by using the posterior samples.

In this work, we use the the transitional Markov Chain Monte Carlo (TMCMC) algorithm developed by Ching and Cheng [47], which can solve the higher-dimensional Bayesian model by updating problems efficiently. Using the conventional MCMC is not practical in the applications of our interest here, since this algorithm is inefficient for generating samples from the posterior PDF especially when the high probability content concentrates in a very small volume of the parameter space. To overcome this problem, TMCMC introduces a "sequence' of intermediate PDFs and the MetropolisHastings $(\mathrm{MH})$ algorithm generates a Markov chain of samples from each intermediate PDF. The more detailed explanation of this scheme and potential issues/improvements are discussed elsewhere [47, 48].

\section{Numerical results}

For each model class, $M_{j}, j=1, \ldots, 4,5000$ samples are generated from the posterior PDF $p\left(\boldsymbol{\theta} \mid D, M_{j}\right)$, using the experimental data and the advanced stochastic simulation method mentioned in the previous section, with 30000 samples in the whole algorithm. Each whole calibration-prediction process took less than an hour on a modern serial Linux machine thanks to the simplicity of the model. In addition, extensive statistical convergence tests using different sample sizes were performed in order to increase our confidence that the PDFs of the parameters and of the quantities of interest are well approximated. The current numerical methodology is very efficient and feasible for various engineering applications (e.g. [25, 26, 40, 43, 49-52]), in addition to a high-dimensional problem [48], where the current method was leveraged to calibrate 31 parameters. The posterior PDFs of $A_{r}, m_{r}$, , for $r=1, \ldots, 6$, are estimated using posterior samples for $\boldsymbol{\theta}$ from $p\left(\boldsymbol{\theta} \mid D, M_{j}\right), j=1, \ldots, 4$, are shown in Figs. 3 (a)-(f), 4 (a)-(f), and 5 (a)-(f), respectively. The vertical 
dotted line in each figure gives the corresponding nominal value as shown in Table 2 except $A_{1}$. Compared with the prior uncertainties in these parameters, it can be seen that their posterior (updated) uncertainties are modified significantly since the data provide information about them.

For cases 1,2, and 3, it can be seen that the nominal values of $m_{1}$ and $\Theta_{1}$ are located near the tail (low probability region) of the corresponding posterior marginal PDFs. It is interesting to note that the values of $A_{1}$ and $m_{1}$ obtained by Szekely et al. [53, 54] and Tabayashi et al. [30] lie inside the high probability region and are similarly probable for these cases. The relatively flat shape of the marginal PDFs of $A_{1}$ and $m_{1}$ indicates that this experiment is not very informative to identify a smaller set of the more probable values of $A_{1}$ and $m_{1}$. The same conclusion applies to the marginal PDFs of $A_{3}$. Case 4 covers the high probability region of case 3 and has similar uncertainty characteristics for the posterior marginal PDFs for $A_{1}$, $A_{3}$, and $m_{3}$ respectively. This shows that the addition of $R 2, R 5$, and $R 6$ as uncertain reactions does not affect significantly the posterior marginal PDFs of these parameters. However, much larger uncertainty in Arrhenius parameters for $R 4$ is observed since the number of uncertain parameters in case 4 is significantly larger. For case 4 , it can be seen that the posterior marginal PDFs of $A_{r}, m_{r}$, and $\Theta_{r}$, for $r=2,5$, and 6 spread over the whole interval covered by the high probability region of the corresponding priors. However, not each value in the interval is equally plausible as assumed in the prior, which implies that this set of experimental data still provides some information for all these uncertain parameters even though the uncertainty still remains significant. Note these experimental data were originally used for determination of $R 3$ [1].

The posterior PDF of the whole output prediction error $r_{\text {total,n }}^{(i)}$ at time $t_{n}$ subjected to the conditions of the $i$-th experiment can be obtained (Fig. 6) as follows using the following approximation:

$$
p(\mathbf{Q} \mid D, M) \approx \frac{1}{K} \sum_{k=1}^{K} \delta\left(\mathbf{Q}-\mathbf{Q}^{(k)}\right)
$$

with $\mathbf{Q}$ being $r_{\text {total, } n}^{(i)}$ and $\mathbf{Q}^{(k)}$ being a sample simulated from $p\left(\mathbf{Q} \mid \boldsymbol{\theta}^{(k)}, D, M\right)$. For $\mathbf{Q}$ equal to $r_{\text {total, },}^{(i)}, p\left(\mathbf{Q} \mid \boldsymbol{\theta}^{(k)}, D, M\right)$ is a log-normal PDF with a median of 1 and $\log$ variance equal to $\left[\sigma_{\text {total }}^{2}\right]^{(k)}$ where $\left[\sigma_{\text {total }}^{2}\right]^{(k)}$ are the $k$-th sample from the corresponding posterior marginal PDFs. Compared with the prior 
uncertainties in $r_{\text {total,n }}^{(i)}$ (Fig. 2), it can be seen that the posterior (updated) uncertainties in $r_{t o t a l, n}^{(i)}$ are reduced since the data provided information about them. For all four model classes, the posterior PDFs of the whole output prediction multiplicative error $r_{\text {total,n }}^{(i)}$ are similar, having median approximately equal to 1 and most of the probability lying in $[0.5,1.6]$ and become narrower though not much with the increase of the number of the uncertain reactions. This can be expected since it is more plausible that the error should be smaller with the increase of the number of the uncertain reactions since there is a larger degree of freedom for the parameters to adjust themselves.

Tables 4, 5, 6, and 7 give the correlation coefficient matrix $R$ and partial correlation coefficient matrix $R_{p}$ for all cases. Only the upper diagonal (the lower diagonal) entries of $R\left(R_{p}\right)$ are shown since it is symmetric. Due to space limitations, for cases 3 and 4 , only parts of the matrices are shown. For case 1, it can be seen from the correlation matrix that there is a very strong correlation (degree of linear dependence) between pairs of Arrhenius model parameters (i.e., $A_{1}, m_{1}$, and $\Theta_{1}$ in this case) and a very weak correlation between an Arrhenius model parameter and its corresponding log error variance parameter (i.e., $\sigma_{\text {total }}^{2}$ ). Strong correlation between two uncertain parameters can be due to the effect of other uncertain parameters. Thus, it is interesting to also look at the degree of linear dependence between two uncertain parameters with the effect of all other uncertain parameters removed by looking at the partial correlation coefficient between them. If the correlation and partial correlation coefficients between a pair of uncertain parameters are very different, the effect of other uncertain parameters is strong in affecting the correlation between them. It can be seen from the partial correlation matrix that with the effect of other uncertain parameters removed, pairs of Arrhenius model parameters are almost perfectly linearly related and an Arrhenius model parameter is almost not linear related to a log error variance parameter. Generally speaking, the pre-factor and activation energy are positively correlated, and the negative-valued power term and activation energy are also positively correlated. This guarantees that the rate coefficient would not become either much higher or much lower than the nominal value. This feature can be seen in the partial correlation matrix. The partial correlation coefficient between $A_{1}$ and $m_{1}$ is close to - 1 implying an almost perfectly negative linear relationship between them. The partial correlation coefficient between $A_{1}$ and $\Theta_{1}$ and between $m_{1}$ and $\Theta_{1}$ is almost 
1 implying an almost perfectly positive linear relationship between these two pairs of uncertain parameters. This linear correlation is consistent with what is seen in the posterior marginal PDFs of $A_{1}$ and $m_{1}$ shown in Fig. 3 (a) and Fig. 4 (a). For case 2, similar observation can be obtained for the Arrhenius model parameters of the same reaction. It is seen that the Arrhenius model parameters of reaction $R 1$ and that of reaction $R 3$ are almost perfectly linearly related (with the effects of all other uncertain parameters removed). These linear relationships are corresponding to the similar shapes of these marginal PDFs shown in Figs. 3-5. Similar to case 1, there is a very weak partial correlation between Arrhenius model parameters. For case 3, we observe that, with the effect of other uncertain parameters removed, $A_{1}$ is strongly negatively correlated with $A_{3}$ and moderately negatively correlated with $A_{4}$, and $A_{3}$ is weakly negatively correlated with $A_{4}$. It is interesting to see that even though the $\mathrm{O}$, which dissociates $\mathrm{HCN}$ through $R 4$, comes mainly from $R 2$ and $R 3, A_{4}$ does not show a strong linear relationship with pre-factors of these reactions. The large difference in the correlations and partial correlations between $A_{1}$ and $A_{3}$ indicates the complex nature of the reaction involving $\mathrm{O}, \mathrm{CN}$, and $\mathrm{HCO}$. The negative partial correlation between $A_{1}$ and $A_{3}$ may be due to the excess of $\mathrm{O}$ generated by the thermal decomposition of $\mathrm{O}_{2}$. Similar to case 2, the partial correlation between an Arrhenius model parameter and its log error variance parameter is very weak. For case 4 , with the effect of other uncertain parameters removed, it can be seen that $A_{1}$ is strongly negatively correlated with $A_{2}$ and $A_{3}$ and weakly correlated with $A_{5}$ and $A_{6}$. Note that the correlation coefficients and partial correlation coefficients between $A_{1}$ and $A_{2}-A_{4}$ are significantly different. This implies that there are significant effects from other reactions. $A_{2}$ is negatively correlated with $A_{3}$ and weakly correlated with $A_{5}$ and $A_{6} . A_{3}$ is weakly negatively correlated with $A_{4}, A_{5}$, and $A_{6}$. $A_{4}$ is moderately negatively correlated with $A_{5}$ and $A_{6}$. It is interesting to see that $R 3$ is not correlated to $R 4-R 6$ as strongly as to $R 1$ and $R 2$, possibly indicating the importance of the $\mathrm{O}_{2}$ dissociation by $H$ and $C N$ from $R 1$. $A_{5}$ is very weakly correlated with $A_{6}$ even though $R 5$ is an essential reaction to create $N$ for $R 6$. This is a good example to show that the correlation matrices cannot capture a nonlinear relationship. Similar to the other cases, in general, there is a very weak partial correlation between an Arrhenius model parameter and its log error variance parameter.

It should be noted that a weak correlation between a pair of uncertain parameters only implies weak linear dependence and does not necessarily imply weak dependence between them unless the uncertain parameters are jointly 
Gaussian (see, for example, the example in Cheung and Beck [40]). Thus to investigate the dependence between different pairs of parameters, looking at the sample plots of pairs of uncertain parameters can be informative. For illustration, the posterior sample plots of some pairs of the components of $\boldsymbol{\theta}$ are shown in Figs. 7 (a) and (b) for cases 1 and 3. These figures show that there is significant dependence between different pairs of Arrhenius parameters but very weak dependence between Arrhenius parameters and the variance parameter. It can be seen that the approach presented in this paper automatically quantifies the complicated probabilistic dependence among all the uncertain parameters, even though a priori the only thing we can often assume is independence among parameters (as in the prior PDFs defined in Table 3), which is mostly incorrect.

Figures 8 (a)-(d) show statistical information of the posterior prediction of $k_{1}$ for all cases based on samples from the posterior PDFs, including the posterior mean, 2.5 percentile and 97.5 percentile for the predicted rate coefficient $k_{1}$ (plotted in common logarithmic scale) of reaction $R 1$ against $10^{4}(\mathrm{~K}) / T$ where $T$ is the temperature. The posterior PDF of the predicted rate coefficient $k_{1}$ at a particular temperature can be obtained using Eq. (13) with $\mathbf{Q}$ being $k_{1}$ at the same temperature and $\mathbf{Q}^{(i)}=k_{1}\left(\boldsymbol{\theta}^{(i)}\right)$. Thus the posterior mean of the predicted $k_{1}$ can be obtained using $E(\mathbf{Q} \mid D, M) \approx$ $\frac{1}{K} \sum_{k=1}^{K} \mathbf{Q}^{(k)}$ and the corresponding posterior percentile can be obtained using the samples $\mathbf{Q}^{(i)}$ 's. The posterior PDF, thus the posterior mean and percentile of the predicted $k_{1}$ are temperature dependent since $k_{1}$ is a function of temperature. For case 1, the nominal value of $k_{1}$ lies within 2.5 percentile and 97.5 percentile (i.e., $95 \%$ confidence interval (CI)) and is very close to the posterior mean in the temperature range of the experimental data. This is because besides $\sigma_{\text {total }}^{2}$, only the Arrhenius model parameters of $R 1$ are considered uncertain while the Arrhenius model parameters of the other 5 reactions are fixed at their nominal values. Note the nominal value of $R 1$ provided by Thielen and Roth was obtained by different experimental setups (i.e., HCN/Ar mixture) in which the experiments were performed in the range of $2210-2480(\mathrm{~K})$. Even though the variation in the temperature range of the experiment is small, their prediction agrees well with the posterior prediction shown here even in the higher temperature range $\geq 4000(\mathrm{~K})$. There is a higher uncertainty in predicting $k_{1}$ at lower temperatures since this is far from the temperature range of the experimental data. Overall, the predicted $95 \% \mathrm{CI}$ is relatively small even though the range of the prior of $A_{1}$ is large, and the magnitude of the uncertainty is consistent with the range 
of comprehensive data provided by the National Institute of Standard and Technology (NIST) [46].

Compared with case 1, case 2, which has the Arrhenius model parameters of $R 3$ as additional uncertain parameters, gives a slightly more uncertainty in $k_{1}$ (see the inserted figure in Fig. 8 (b)). Nominal values of $k_{1}$ no longer lie within the $95 \%$ CI bounded by the posterior 2.5 percentile and 97.5 percentile. Within the temperature range of the experimental data, the 2.5 percentile and 97.5 percentile can differ by about a factor 3 , while the nominal values and the posterior mean of $k_{1}$ can differ by about a factor 2 . Case 2 assumes reactions $R 1$ and $R 3$ are unknown as in the work of Thielen and Roth. From the difference in the results between this case and other cases, one can see that the choice of uncertain reactions is important. In this system, the major active species are $\mathrm{HCN}$ and $\mathrm{O}_{2}$ at the early stage of the reaction. $R 1$ and $R 3$ are tightly coupled through CN. It is worth noting that reported $k_{1}$ values from past studies differ by up to a factor of 5 [46]. Similar to case 1 , there is a higher uncertainty in predicting $k_{1}$ at the lower temperature region. It can be seen that the results for the prediction of $k_{1}$ for case 3 and case 4 are similar to case 2 except that the uncertainty increases with the number of uncertain reactions. This shows that having the Arrhenius model parameters of $R 2, R 4, R 5$ and $R 6$ as additional uncertain parameters does not have a significant effect on the high probability region in the prediction of $k_{1}$ compared with the cases obtained based on the nominal values of those reactions. This can be understood by the fact that the major path of the destruction process is controlled by $R 1$ and the concentration of $\mathrm{O}$ is not significant at the early stage. Within the temperature range of the experimental data, the results for the prediction of $k_{1}$ for only case 1 show that the nominal values stays inside the $95 \%$ CI.

Figure 9 shows statistical information of the posterior prediction of $k_{2}$ for case 4 . Compared with $k_{1}$, the $95 \%$ CI is quite wide even within the temperature range of experimental data showing that the experimental data provide only some information for this rate coefficient. Also, there is a high probability that the nominal values of $k_{2}$ differ from the posterior mean by as much as a factor 5 . In fact, even though there are numerous past studies for $R 2$, the uncertainty of $k_{2}$ still remains large. [46]

Figures 10 (a)-(c) show statistical information of the posterior prediction of $k_{3}$ for cases 2, 3 and 4 . The results of cases 2 and 4 are quite similar. This shows that having $R_{2}, R_{4}, R_{5}$ and $R_{6}$ as additional uncertain reactions does not significantly affect the uncertainty characteristics in the prediction of $k_{3}$. 
Within the temperature range of the experimental data, the 2.5 percentile and 97.5 percentile can differ by a factor 3 , and the nominal values and the posterior mean of $k_{3}$ can differ by as much as a factor 2. As compared with the other reported values in addition to [8] and [55], it can be seen that the nominal value is possibly underestimated by a factor of 2 [46]. The results for the prediction of $k_{3}$ for case 3 are slightly different from those of cases 2 and 4 . The $95 \%$ CI remains wide and shows very weak temperature dependence over the wide range of temperature. Past studies showed the slight temperature dependence of $k_{3}[46]$.

Figures 11 (a) and (b) show statistical information of the posterior prediction of $k_{4}$ for cases 3 and 4 . It can be seen that the increase of the number of uncertain reactions (i.e., $R 2, R 5$ and $R 6$ ) does not cause the posterior mean to change significantly but increases the uncertainty (i.e., wider $95 \%$ CI) within the temperature range of experimental data and changes the uncertainty characteristics more significantly at lower temperatures. Given the current experimental data and kinetics, there is a significant uncertainty in the predicted $k_{4}$. For both cases, the nominal values of $k_{4}$ stay outside the 95\% CI within the temperature range of experimental data, but stay inside the CI at lower temperatures since the CI is wide enough at lower temperatures. It is interesting to note that the posterior mean of $k_{4}$ for case 4 is very close to the nominal value at lower temperatures. The suggested rates of $R 4$ by Tsang and Herron [10] are $2.2 \times 10^{8} \mathrm{~cm}^{3} \mathrm{~mol}^{-1} \mathrm{~s}^{-1}$ at $1000 \mathrm{~K}$ and $2.9 \times 10^{12} \mathrm{~cm}^{3} \mathrm{~mol}^{-1} \mathrm{~s}^{-1}$ at $2500 \mathrm{~K}$. The former value can differ from the posterior predicted values by as much as two orders of magnitude for both cases. Even though Thielen and Roth [1] suggest almost the same values for $\Theta_{r}$ in the reactions which involve the decomposition of $\mathrm{HCN}$ by $\mathrm{O}$ (i.e., $R 4$, $R 9$, and $R 10$ ), the above observations lead us to think that an alternative reaction mechanism should be explored.

Figure 12 shows statistical information of the posterior prediction of $k_{5}$ for case 4 . The nominal values of $k_{5}$ lie between the $95 \%$ CI in the low temperature region, but not within the temperature range of experimental data. There is a significant uncertainty in the predicted $k_{5}$. According to the published data, the rate of $k_{5}$ still remains quite uncertain due to the uncertainty in the activation energy (e.g., $k_{5}: 6 \times 10^{9} \mathrm{~cm}^{3} \mathrm{~mol}^{-1} \mathrm{~s}^{-1}[56]$ and $1.2 \times 10^{11} \mathrm{~cm}^{3} \mathrm{~mol}^{-1} \mathrm{~s}^{-1}$ at $\left.2600 \mathrm{~K}[57]\right)$.

Figure 13 shows statistical information of the posterior prediction of $k_{6}$ for case 4. Even though the high probability region of the posterior PDF of $A_{6}$ (see Fig. 3 (f) covers a wide interval, the resulting prediction of $k_{6}$ 
has much smaller uncertainty particularly within the temperature range of experimental data because data provide information about the probabilistic dependence of $A_{6}$ with $m_{6}$ and $\Theta_{6}$ and other uncertain parameters, which constrains the high probability region of $k_{6}$. The nominal values stay close to the posterior prediction especially in the high temperature region. In the past, because of the significance of this reaction for the understanding of the mechanism of NOx, this reaction was intensively investigated especially in the low temperature region $(\leq 1000 \mathrm{~K})$ [46], but not much in the high temperature region [57]. In this high temperature experiment, the main destruction mechanism of $\mathrm{O}_{2}$ are $R 2$ and $R 3$, and thus the amount of uncertainty which can be reduced by this experimental data for reaction $R 6$ seems to be limited.

Figure 14 shows statistical information of the posterior prediction of $k_{18} / k_{5}$ for case 4 . For the rate coefficient $k_{18}$ of $R 18$, its nominal value is used since its uncertainty has not been quantified here. The three error bars in this figure are the results reported in a previous study [34]. Given the current set of data, there is a significant uncertainty in the predicted $k_{18} / k_{5}$ that can differ by as much as 7 orders of magnitude depending on temperature. In this study, the experimental data set is not used for quantifying the uncertainty in $k_{18}$ since only the six reactions $R 1-R 6$ are considered. If the uncertainty in $k_{18}$ is also quantified (this will be left for future studies), the result is expected to change. From this observation, it can be deduced that rate coefficients derived from one choice of reduced chemical kinetics and quantified using one set of data may not work with the desired tolerances of accuracy in a different chemical system.

For the purpose of sanity checking, we compared the posterior prediction of concentrations subjected to the same set of experimental conditions with the experimental data that are used for the calibration procedure. Figures 15 (a)-(d) show statistical information of the posterior prediction of the concentration of nitrogen atom. The initial conditions are: $T=2600 \mathrm{~K}$, $[\mathrm{Ar}]=8.36 \times 10^{6} \mathrm{~mol} \mathrm{~cm}^{-3}, \mathrm{HCN}$ and $\mathrm{O}_{2}$ concentration of $50 \mathrm{ppm}$ each. For all cases, the experimental data lie between $95 \%$ confidence interval which means the experimental data lie inside the high probability region of the posterior prediction obtained by the Bayesian approach. It can be seen that there is a 0.95 probability that at each time, the predicted concentration of nitrogen atom is within $\pm 30 \%$ of the posterior mean. For reference, recall that the reported experimental uncertainty for concentration measurement of $\mathrm{N}$ is $\pm 25 \%$. For comparison, the concentration profiles obtained by using 
the nominal values are also shown. However, it is worth mentioning that the nominal profiles fit to the experimental data well initially and then start to diverge from the data.

Figures 16 (a)-(d) show statistical information of the posterior prediction of the concentration of oxygen atom. The initial conditions are: the temperature is $2826 \mathrm{~K}$; the Argon concentration is $7.27 \times 10^{6} \mathrm{~mol} \mathrm{~cm}^{-3}$; the $\mathrm{HCN}$ concentration and the $\mathrm{O}_{2}$ concentration are $50 \mathrm{ppm}$ each. For all cases, the experimental data all lie between 2.5 percentile and 97.5 percentile which means the experimental data lie inside the high probability region of the posterior prediction. It can be also seen that there is a 0.95 probability that at each time, the predicted concentration of oxygen atom is within $\pm 30 \%$ of the posterior mean. For reference, recall that the reported experimental uncertainty for the concentration measurement of $\mathrm{O}$ is $\pm 15 \%$. The posterior mean stays close to the experimental data for all cases.

In summary, all the proposed model classes are able to predict the concentration of selected species with quantified uncertainty, which covers the data with a reasonable tolerance. It can also be seen that the predicted uncertainty is more than the reported experimental uncertainty in the concentration measurement. Finally, as seen in Figs 15 (a)-(d) and Figs. 16 (a)-(d), the nominal rate coefficients which have good agreement with the experimental data give one of the plausible solutions, but not the only ones. From this exercise, we derive a conclusion that to reproduce this type of the data (e.g., [58]) can be misleading in context of checking the validity of chemical reaction mechanism, especially when taking account of uncertainties.

\section{Conclusion}

In this paper, a robust stochastic system model based Bayesian approach used the experimental data presented in [1] to quantify the uncertainties in the modeling of the $\mathrm{HCN} / \mathrm{O}_{2} / \mathrm{Ar}$ mixture kinetics proposed by Thielen and Roth [1]. The uncertainties in the rate coefficients and the time evolution of concentration for the involved species were also determined. The uncertainty in the experimental measurement error and physical modeling error are also quantified. The results show that there can be significant statistical dependence among the modeling parameters and rate coefficients, and the uncertainties in the quantities of interest are temperature dependent. For a better understanding of the complex nature of reaction $R 3$ as mentioned in Sec 1, it is necessary to perform more experiments to obtain information 
about this reaction or to construct a new mechanism aiming specifically at obtaining this rate coefficient using the current experimental data. This will be left for a future work.

In a true chemical kinetics system studied here, there is both a system identification and prediction problem coupled with limited experimental data. An important contribution of adopting the stochastic system model based Bayesian approach is that it enables one to quantify the relative plausibility of multiple stochastic solutions due to incomplete system knowledge and to obtain robust solutions. Last but not least, the uncertainties in both the uncertain parameters and the quantities of interest are shown to be quite sensitive to the number of uncertain parameters and the number of uncertain reactions. The number of uncertain reactions can be viewed as a form of model structure uncertainty which leads one to consider how many reactions should be modeled. Cheung and Beck $([40,41])$ propose a robust multiple-stochastic system model based Bayesian approach to obtain a more robust uncertain quantification and prediction of quantities of interest. This approach proposes to solve uncertainty quantification problem considering multiple hypotheses involving stochastic models that considers uncertainty beyond parametric uncertainty and can be applied to the problem of how

many uncertain reactions to consider. The results applying this approach in the context of chemical kinetics will be presented in our future publications. We are currently applying the method presented in this paper to a chemical system involving many reactions of the order of hundred.

\section{Acknowledgement}

We are very grateful to Professor Philip L. Varghese at the University of Texas at Austin for his helpful discussions and comments on the manuscript. This material is based upon work supported by the Department of Energy [National Nuclear Security Administration] under Award Number [DE-FC5208NA28615]. 


\section{Appendices}

\section{A. Stochastic system model based Bayesian approach for quanti- fying uncertainties}

\section{A.1. General form of input-output predictive models}

One important special case of these models is the stochastic model of dynamical systems (e.g., $[59,60])$ :

$$
\begin{aligned}
\dot{\mathbf{x}}(t) & =\dot{\mathbf{x}}(0) \\
\dot{\mathbf{x}}(t) & =\widetilde{\mathbf{f}}(x(t), u(t), \boldsymbol{\gamma}(t), t ; \boldsymbol{\theta}) \\
\mathbf{y}(t) & =\widetilde{\mathbf{h}}(\mathbf{x}(t), u(t), \boldsymbol{\varepsilon}(t), t ; \boldsymbol{\theta})
\end{aligned}
$$

Here, Eq. (A.1) represents a continuous time state evolution equation, which can be viewed as a special case of the first equation in Eq. (5), and Eq. (A.2) is a continuous time observation output equation which can be viewed as a special case of the combination of the second and third equations in Eq. (5).

In a discrete case which we consider above (see Eq. (6)), the above equations take the form of the following:

$$
\begin{gathered}
\mathbf{x}_{n}=\mathbf{f}_{n-1}\left(\mathbf{x}_{n-1}, \mathbf{u}_{n-1}, \boldsymbol{\gamma}_{n-1} ; \boldsymbol{\theta}\right) \\
\mathbf{y}_{n}=\mathbf{h}_{n}\left(\mathbf{x}_{n}, \mathbf{u}_{n}, \boldsymbol{\varepsilon}_{n} ; \boldsymbol{\theta}\right)
\end{gathered}
$$

where $\mathbf{x}_{n}\left(=\mathbf{x}\left(t_{n}\right)\right), \mathbf{u}_{n}\left(=\mathbf{u}\left(t_{n}\right)\right), \mathbf{y}_{n}\left(=\mathbf{y}\left(t_{n}\right)\right), \boldsymbol{\gamma}_{n}\left(=\gamma\left(t_{n}\right)\right)$, and $\boldsymbol{\varepsilon}_{n}\left(=\boldsymbol{\varepsilon}\left(t_{n}\right)\right)$ denote the model state, the system input, the observed system output, the uncertain disturbances (e.g., process noise, or dynamic model equation error), and the output equation errors (which can include measurement errors) at the $n$-th time instant, $t_{n}, n=0, . ., N_{T}$. The probability models (which have uncertain model parameters $) p\left(\left[\boldsymbol{\gamma}_{1}^{T} \boldsymbol{\gamma}_{2}^{T} \ldots \boldsymbol{\gamma}_{N_{T}}^{T}\right]^{T} \mid \boldsymbol{\theta}, M\right)$ and $p\left(\left[\varepsilon_{1}^{T} \boldsymbol{\varepsilon}_{2}^{T} \ldots\right.\right.$ $\left.\left.\boldsymbol{\varepsilon}_{N_{T}}^{T}\right]^{T} \mid \boldsymbol{\theta}, M\right)$ for the $\boldsymbol{\gamma}_{n}$ 's and $\boldsymbol{\varepsilon}_{n}$ 's are chosen using the Principle of Maximum Information Entropy for this study [39]. The two fundamental system probability models given in Eqs. (A.3) and (A.4), along with the specification of the PDF $p\left(\mathbf{x}_{0} \mid \boldsymbol{\theta}, M\right)$ for the initial states, completely define the stochastic dynamics of the system. $\boldsymbol{\theta}$ is a vector denoting all the uncertain parameters for the problem. 
[1] K. Thielen, P. Roth, Resonance absorption measurements of N, O, and $\mathrm{H}$ atoms in shock heated $\mathrm{HCN} / \mathrm{O}_{2} /$ Ar mixtures, Combustion and Flame 69 (1987) 141-154.

[2] K. H. Becker, R. Kurtenbach, F. Schmidt, P. Wiesen, Kinetics of the $\mathrm{NCO}$ radical reacting with atoms and selected molecules, Combustion and Flame 120 (2000) 570-577.

[3] C. J. Cobos, Statistical adiabatic channel model rate constants for the reaction $\mathrm{O}+\mathrm{CN} \rightarrow \mathrm{CO}+\mathrm{N}\left({ }^{2} D\right)$ at $300-5000 \mathrm{~K}$, Reaction Kinetics and Catalysis Letters 57 (1996) 43-47.

[4] T. Faravelli, A. Frassoldati, E. Ranzi, Kinetic modeling of the interactions between NO and hydrocarbons in the oxidation of hydrocarbons at low temperatures, Combustion and Flame 132 (2003) 188-207.

[5] A. Frassoldati, T. Faravelli, E. Ranzi, Kinetic modeling of the interactions between NO and hydrocarbons at high temperature, Combustion and Flame 135 (2003) 97-112.

[6] D. Lindackersa, M. Burmeister, P. Roth, High-temperature kinetics of the reaction $\mathrm{CN}+\mathrm{CO}_{2}$, Combustion and Flame 81 (1990) 251-259.

[7] M. Y. Louge, R. K. Hanson, High temperature kinetics of NCO, Combustion and Flame 58 (1984) 291-300.

[8] J. N. Mulvihill, L. F. Phillips, Breakdown of cyanogen in fuel-rich $\mathrm{H}_{2} / \mathrm{N}_{2} / \mathrm{O}_{2}$ flames, 15th Symposium (International) on Combustion, The Combustion Institute (1975) 1113-1122.

[9] P. Roth, M. Y. Louge, R. K. Hanson, O- and N- atom measurements in high temperature $\mathrm{C}_{2} \mathrm{~N}_{2}+\mathrm{O}$ kinetics, Combustion and Flame 64 (1986) 167-176.

[10] W. Tsang, J. T. Herron, Chemical kinetic data base for propellant combustion. I. reactions involving $\mathrm{NO}, \mathrm{NO}_{2}, \mathrm{HNO}, \mathrm{HNO}_{2}, \mathrm{HCN}$, and $\mathrm{N}_{2} \mathrm{O}$, Journal of Physical and Chemical Reference Data 20 (1991) 609-663.

[11] V. Vasudevan, D. F. Davidson, R. K. Hanson, Direct measurements of the reaction $\mathrm{OH}+\mathrm{CH}_{2} \mathrm{O} \rightarrow \mathrm{HCO}+\mathrm{H}_{2} \mathrm{O}$ at high temperatures, International Journal of Chemical Kinetics 37 (2004) 98-109. 
[12] E. Ranzi, M. Dente, A. Goldaniga, G. Bozzano, T. Faravelli, Lumping procedures in detailed kinetic modeling of gasification, pyrolysis, partial oxidation and combustion of hydrocarbon mixtures, Progress in Energy and Combustion Science (2001) 99-139.

[13] S. Javoy, R. Mevel, C. E. Paillard, A study of $\mathrm{N}_{2} \mathrm{O}$ decomposition rate constant at high temperature: Application to the reduction of nitrous oxide by hydrogen, International Journal of Chemical Kinetics 41 (2009) $357-375$.

[14] M. T. Reagan, H. N. Najm, R. G. Ghanem, O. M. Knio, Uncertainty quantification in reacting-flow simulations through non-intrusive spectral projection, Combustion and Flame 132 (2003) 545-555.

[15] M. T. Reagan, H. N. Najm, P. P. Pébay, O. M. Knio, R. G. Ghanem, Quantifying uncertainty in chemical systems modeling, International Journal of Chemical Kinetics 37 (2005) 368-382.

[16] I. G. Zsély, J. Zádor, T. Turányi, Uncertainty analysis of NO production during methane combustion, International Journal of Chemical Kinetics 40 (2008) 754-768.

[17] T. Russi, A. Packard, R. Feeley, M. Frenklach, Sensitivity analysis of uncertainty in model prediction, The Journal of Physical Chemistry A 112 (2008) 2579-2588.

[18] D. A. Sheen, X. You, H. Wang, T. Løvas, Spectral uncertainty quantification, propagation and optimization of a detailed kinetic model for ethylene combustion, Proceedings of the Combustion Institute 32 (2009) $535-542$.

[19] D. A. Sheen, H. Wang, The method of uncertainty quantification and minimization using polynomial chaos expansions, Combustino and Flame 158 (2011) 2358-2374.

[20] S. G. Davis, A. V. Joshi, H. Wang, F. Egolfopoulos, An optimized kinetic model of $\mathrm{H}_{2} / \mathrm{CO}$ combustion, Proceedings of the Combustion Institute 30 (2005) 1283-1292.

[21] L. Elliott, D. B. Ingham, A. G. Kyne, N. S. Mera, M. Pourkashanian, C. W. Wilson, A novel approach to the optimization of reaction rate 
parameters for methane combustion using multi-objective genetic algorithms, Journal of Engineering for Gas Turbines and Power 126 (2004) 455-464.

[22] A. Bardow, W. Marquardt, Incremental and simultaneous identication of reaction kinetics: methods and comparison, Chemical Engineering Science (2004) 2673-2684.

[23] M. Brendel, D. Bonvin, W. Marquardt, Incremental identification of kinetic models for homogeneous reaction systems, Chemical Engineering Science (2006) 5404-5420.

[24] G. Reinisch, K. Miki, G. L. Vignoles, B. M. Wong, C. Simmons, An efficient and accurate formalism for the treatment of large amplitude intramolecular motion, Journal of Chemical Theory and Computation 8 (2012) 2713-2724.

[25] K. Miki, E. E. Prudencio, S. H. Cheung, G. Terejanu, Using Bayesian analysis to quantify uncertainties in the $\mathrm{H}+\mathrm{O}_{2} \rightarrow \mathrm{OH}+\mathrm{O}$ reaction, Combustion and Flame 260 (2013) 861-869.

[26] K. Miki, S. H. Cheung, E. E. Prudencio, P. Varghese, Bayesian uncertainty quantification of recent shock tube determinations of the rate coefficient of reaction $\mathrm{H}+\mathrm{O}_{2} \rightarrow \mathrm{OH}+\mathrm{O}$, International Journal of Chemical Kinetics 44 (2012) 586-597.

[27] J. Prager, H. Najm, K. Sargsyan, C. Safta, W. Pitz, Uncertainty quantification of reaction mechanisms accounting for correlations introduced by rate rules and fitted arrhenius parameters, Combustion and Flame 160 (2013) 1583-1593.

[28] T. Nagy, T. Turányi, Uncertainty of arrhenius parameters, International Journal of Chemical Kinetics 43 (2011) 359-378.

[29] A. Szekely, R. K. Hanson, C. T. Bowman, Thermal decomposition of hydrogen cyanide behind incident shock waves, The Journal of Physical Chemistry 88 (1984) 666-668.

[30] K. Tabayashi, T. Fueno, K. Takase, D. Kajimoto, K. Okada, Thermal decomposition of hydrogen cyanide in shock waves, Bulletin of the Chemical Society of Japan 50 (1977) 1754-1757. 
[31] P. Roth, T. Just, Messungen zum thermischen zerfall von HCN hinter sto $\beta$ wellen, Berichte der Bunsengesellschaft für Physikalische Chemie 80 (1976) 171-179.

[32] P. Roth, R. Löhr, H. D. Hermanns, Sto $\beta$ wellenmessugen zur kinetic der reacrtion $\mathrm{HCN}+\mathrm{O}$, Berichte der Bunsengesellschaft für Physikalische Chemie 84 (1980) 835-840.

[33] D. L. Baulch, C. C. J., R. A. Cox, P. Frank, G. Hayman, T. Just, J. A. Kerr, T. Murells, M. J. Pilling, J. Troe, R. Walker, J. Warnatz, Evaluated kinetic data for combustion modeling: Supplement i., Journal of Physical and Chemical Reference Data 23 (1994) 847-1033.

[34] M. Y. Louge, R. K. Hanson, Shock tube study of NCO kinetics, 20th Symposium (International) on Combustion, The Combustion Institute (1984) 665-675.

[35] K. Thielen, P. Roth, Sto $\beta$ wellenuntersuchungen zum start der reaktion $\mathrm{CO}+\mathrm{O}_{2}$, Berichte der Bunsengesellschaft für Physikalische Chemie 87 (1983) 920-925.

[36] R. A. Perry, C. F. Melius, The rate and mechanism of the reaction of HCN with oxygen atoms over the temperature range $540900 \mathrm{k}$, 20th Symposium (International) on Combustion, The Combustion Institute (1984) 639-646.

[37] J. L. Beck, L. S. Katafygiotis, Updating of a model and its uncertainties utilizing dynamic test data, Proc. 1st International Conference on Computational Stochastic Mechanics (1991) 125-136.

[38] J. L. Beck, L. S. Katafygiotis, Updating models and their uncertainties. I: Bayesian statistical framework, ASCE Journal of Engineering Mechanics 124 (1998) 455-461.

[39] E. T. Jaynes, Probability Theory: The Logic of Science, Cambridge University Press, 2003.

[40] S. H. Cheung, J. L. Beck, Calculation of posterior probabilities for Bayesian model class assessment and averaging from posterior samples based on dynamic system data, Computer Methods in Applied Mechanics and Engineering (2010) 304-321. 
[41] J. L. Beck, S. H. Cheung, Probability Logic, Model Uncertainty and Robust Predictive System Analysis, Proc. International Conference on Structural Safety and Reliability, Osaka, Japan, September, 2009.

[42] S. H. Cheung, J. L. Beck, Comparison of different model classes for Bayesian updating and robust predictions using stochastic state-space system models., Proc. 10th International Conference on Structural Safety and Reliability, (ICOSSAR09), Osaka, Japan, September 13-17, 2009.

[43] K. Miki, M. Panesi, E. E. Prudencio, S. Prudhomme, Probabilistic models and uncertainty quantification for the ionization reaction rate of atomic nitrogen, Journal of Computational Physics 231 (2012) 38713886 .

[44] P. Congdon, Bayesian Statistical Modeling, Wiley, New York, 2002.

[45] D. Spiegelhalter, N. Best, B. Carlin, A. van der Linde, Bayesian measures of model complexity and fit, Journal of the Royal Statistical Society Series B: Statistical Methodology 64 (2002) 583-639.

[46] J. A. Manion, R. E. Huie, R. D. Levin, D. R. Burgess Jr., V. L. Orkin, W. Tsang, W. S. McGivern, J. W. Hudgens, V. D. Knyazev, D. B. Atkinson, E. Chai, A. M. Tereza, C.-Y. Lin, T. C. Allison, W. G. Mallard, F. Westley, J. T. Herron, R. F. Hampson, D. H. Frizzell, Nist chemical kinetics database, nist standard reference database 17 , version 7.0 (web version), release 1.4.3, data version 2008.12,National Institute of Standards and Technology, Gaithersburg, Maryland, 20899-8320. Web address: http://kinetics.nist.gov/.

[47] J. Ching, Y. J. Chen, Transitional markov chain monte carlo method for Bayesian model updating, model class selection and model averaging, ASCE Journal of Engineering Mechanics 133 (2007) 816-832.

[48] S. H. Cheung, J. L. Beck, Bayesian model updating using hybrid monte carlo simulation with application to structural dynamic models with many uncertain parameter, Journal of Engineering Mechanics 135 (2009) $243-255$. 
[49] S. H. Cheung, T. A. Oliver, E. E. Prudencio, S. Prudhomme, R. Moser, Bayesian uncertainty analysis with applications to turbulence modeling, Reliability Engineering and System Safety 96 (2011) 1137-1149.

[50] R. R. Upadhyay, K. Miki, O. Ezekoye, J. Marschall, Uncertainty quantification of a graphite nitridation experiment using a Bayesian approach, Experimental Thermal and Fluid Science 35 (2011) 1588-1599.

[51] M. Panesi, K. Miki, S. Prudhomme, A. Brandis, On the assessment of a Bayesian validation methodology for data reduction models relevant to shock tube experiments, Computer Methods in Applied Mechanics and Engineering 213 (2011) 383-398.

[52] K. Miki, M. Panesi, E. E. Prudencio, S. Prudhomme, Estimation of the nitrogen ionization reaction rate using EAST data and Bayesian model analysis, Physics of Plasmas 19 (2012) 023507.

[53] A. Szekely, R. K. Hanson, C. T. Bowman, Shock tubes and waves, Proc. the 13th International symposium on Shock Tubes and Waves (1982) 617-619State University of New York, Press, Albany New York.

[54] A. Szekely, R. K. Hanson, C. T. Bowman, Shock tube study of the thermal decomposition of cyanogen, The Journal of Chemical Physics 80 (1984) 4982-4985.

[55] E. A. Albers, K. Hoyermann, H. Schacke, K. J. Schmatjko, H. G. Wagner, J. Wolfrum, Absolute rate coefficients for the reaction of $\mathrm{H}-$ atoms with $\mathrm{N}_{2} \mathrm{O}$ and some reactions of CN radicals, 15th Symposium (International) on Combustion, The Combustion Institute (1975) 765-773.

[56] J. D. Mertens, R. K. Hanson, A shock tube study of $\mathrm{H}+\mathrm{HNCO} \rightarrow \mathrm{H}_{2}+$ $\mathrm{NCO}$ and the thermal decomposition of NCO, 26th Symposium (International) on Combustion, The Combustion Institute 26 (1996) 551-558.

[57] D. L. Baulch, C. T. Bowman, C. C. J., R. A. Cox, T. Just, J. A. Kerr, M. J. Pilling, D. Stocker, J. Troe, W. Tsang, R. Walker, J. Warnatz, Evaluated kinetic data for combustion modeling: Supplement ii., Journal of Physical and Chemical Reference Data 34 (2005) 757-1397. 
[58] P. Dagaut, P. Glarborg, M. U. Alzueta, The oxidation of hydrogen cyanide and related chemistry, Progress in Energy and Combustion Science 34 (2008) 1-46.

[59] S. H. Cheung, J. L. Beck, Updating Reliability of Monitored Nonlinear Structural Dynamic Systems Using Real-time Data, Proc. Inaugural International Conference of the Engineering Mechanics Institute (EM08), University of Minnesota, Minneapolis, Minnesota, USA, May 18-21, 2008.

[60] J. Ching, J. L. Beck, K. A. Porter, Bayesian state and parameter estimation of uncertain dynamical systems, Probabilistic Engineering Mechanics 21 (2006) 81-96. 
Table 1: Results of Arrhenius coefficients of $R 1$ in past studies

\begin{tabular}{c|ccc}
\hline Reference & $10^{A}$ & $m$ & $\Theta$ \\
\hline Szekely et al. [53] & $4.0 \times 10^{14}$ & 0.0 & 44740 \\
Szekely et al. [29] & $1.0 \times 10^{16}$ & 0.0 & 54650 \\
Roth and Just [31] & $1.2 \times 10^{16}$ & 0.0 & 50196 \\
Tabayashi et al. [30] & $5.7 \times 10^{16}$ & 0.0 & 59060 \\
Thielen et al. [1] & $3.0 \times 10^{22}$ & -1.58 & 61435 \\
\hline
\end{tabular}


Table 2: List of reactions and reference rate coefficients. All the rate coefficients are in the form $10^{A} T^{m} \exp \left(-\frac{\Theta}{T}\right)$ except reaction 22 which has the rate in the form $10^{A} T^{m} \exp \left(-\frac{T}{\Theta}\right)[1]$

\begin{tabular}{|c|c|c|c|c|}
\hline Index & Reaction & $A$ & $m$ & $\Theta$ \\
\hline$R 1$ & $\mathrm{HCN}+\mathrm{Ar} \rightarrow \mathrm{H}+\mathrm{CN}+\mathrm{Ar}$ & 22.477 & -1.58 & 61435 \\
\hline$R 2$ & $\mathrm{O}_{2}+\mathrm{H} \rightarrow \mathrm{OH}+\mathrm{O}$ & 14.369 & 0.0 & 8696 \\
\hline$R 3$ & $\mathrm{O}_{2}+\mathrm{CN} \rightarrow \mathrm{NCO}+\mathrm{O}$ & 12.690 & 0.0 & 0 \\
\hline$R 4$ & $\mathrm{HCN}+\mathrm{O} \rightarrow \mathrm{NCO}+\mathrm{H}$ & 13.860 & 0.0 & 7460 \\
\hline$R 5$ & $\mathrm{NCO}+\mathrm{Ar} \rightarrow \mathrm{CO}+\mathrm{N}+\mathrm{Ar}$ & 16.799 & -0.5 & 24000 \\
\hline$R 6$ & $\mathrm{O}_{2}+\mathrm{N} \rightarrow \mathrm{NO}+\mathrm{O}$ & 10.235 & 1.0 & 4650 \\
\hline$R 7$ & $\mathrm{HCN}+\mathrm{H} \rightarrow \mathrm{CN}+\mathrm{H}_{2}$ & 14.563 & 0.0 & 12330 \\
\hline$R 8$ & $\mathrm{HCN}+\mathrm{CN} \rightarrow \mathrm{C}_{2} \mathrm{~N}_{2}+\mathrm{H}$ & 12.707 & 0.0 & 0 \\
\hline$R 9$ & $\mathrm{HCN}+\mathrm{O} \rightarrow \mathrm{NH}+\mathrm{CO}$ & 13.338 & 0.0 & 7440 \\
\hline$R 10$ & $\mathrm{HCN}+\mathrm{O} \rightarrow \mathrm{CN}+\mathrm{OH}$ & 13.338 & 0.0 & 7440 \\
\hline$R 11$ & $\mathrm{HCN}+\mathrm{OH} \rightarrow \mathrm{CN}+\mathrm{H}_{2} \mathrm{O}$ & 13.161 & 0.0 & 5500 \\
\hline$R 12$ & $\mathrm{NO}+\mathrm{N} \rightarrow \mathrm{N}_{2}+\mathrm{O}$ & 13.380 & 0.0 & 0 \\
\hline$R 13$ & $\mathrm{NO}+\mathrm{H} \rightarrow \mathrm{OH}+\mathrm{N}$ & 14.230 & 0.0 & 24560 \\
\hline$R 14$ & $\mathrm{CN}+\mathrm{O} \rightarrow \mathrm{CO}+\mathrm{N}$ & 13.301 & 0.0 & 210 \\
\hline$R 15$ & $\mathrm{CN}+\mathrm{N} \rightarrow \mathrm{N}_{2}+\mathrm{C}$ & 14.641 & 0.0 & 4530 \\
\hline$R 16$ & $\mathrm{CN}+\mathrm{OH} \rightarrow \mathrm{NCO}+\mathrm{H}$ & 13.255 & 0.0 & 0 \\
\hline$R 17$ & $\mathrm{NCO}+\mathrm{H} \rightarrow \mathrm{CO}+\mathrm{NH}$ & 14.017 & 0.0 & 1000 \\
\hline$R 18$ & $\mathrm{NCO}+\mathrm{O} \rightarrow \mathrm{CO}+\mathrm{NO}$ & 13.748 & 0.0 & 0 \\
\hline$R 19$ & $\mathrm{NCO}+\mathrm{N} \rightarrow \mathrm{N}_{2}+\mathrm{CO}$ & 13.301 & 0.0 & 0 \\
\hline$R 20$ & $\mathrm{NCO}+\mathrm{N} \rightarrow \mathrm{CN}+\mathrm{NO}$ & 14.658 & 0.0 & 5530 \\
\hline$R 21$ & $\mathrm{CO}+\mathrm{O}_{2} \rightarrow \mathrm{CO}_{2}+\mathrm{O}$ & 13.699 & 0.0 & 31800 \\
\hline$R 22$ & $\mathrm{CO}+\mathrm{OH} \rightarrow \mathrm{CO}_{2}+\mathrm{H}$ & 10.602 & 0.0 & $775^{*}$ \\
\hline$R 23$ & $\mathrm{NH}+\mathrm{OH} \rightarrow \mathrm{H}_{2} \mathrm{O}+\mathrm{N}$ & 11.699 & 0.5 & 1000 \\
\hline$R 24$ & $\mathrm{NH}+\mathrm{O} \rightarrow \mathrm{NO}+\mathrm{H}$ & 11.799 & 0.5 & 0 \\
\hline$R 25$ & $\mathrm{NH}+\mathrm{H} \rightarrow \mathrm{N}+\mathrm{H}_{2}$ & 13.699 & 0.0 & 1000 \\
\hline$R 26$ & $\mathrm{H}_{2}+\mathrm{O} \rightarrow \mathrm{OH}+\mathrm{H}$ & 14.357 & 0.0 & 6920 \\
\hline$R 27$ & $\mathrm{H}_{2}+\mathrm{OH} \rightarrow \mathrm{H}_{2} \mathrm{O}+\mathrm{H}$ & 13.673 & 0.0 & 3067 \\
\hline$R 28$ & $\mathrm{C}_{2} \mathrm{~N}_{2}+\mathrm{O} \rightarrow \mathrm{CN}+\mathrm{NCO}$ & 12.659 & 0.0 & 4440 \\
\hline$R 29$ & $\mathrm{C}_{2} \mathrm{~N}_{2}+\mathrm{O} \rightarrow \mathrm{CO}+\mathrm{NCN}$ & 14.139 & 0.0 & 7540 \\
\hline$R 30$ & $\mathrm{H}_{2} \mathrm{O}+\mathrm{O} \rightarrow \mathrm{OH}+\mathrm{OH}$ & 13.819 & 0.0 & 9240 \\
\hline$R 31$ & $\mathrm{O}_{2}+\mathrm{Ar} \rightarrow \mathrm{O}+\mathrm{O}+\mathrm{Ar}$ & 18.255 & -1.0 & 59380 \\
\hline$R 32$ & $\mathrm{H}_{2}+\mathrm{Ar} \rightarrow \mathrm{H}+\mathrm{H}+\mathrm{Ar}$ & 14.342 & 0.0 & 48300 \\
\hline$R 33$ & $\mathrm{C}_{2} \mathrm{~N}_{2}+\mathrm{Ar} \rightarrow \mathrm{CN}+\mathrm{CN}+\mathrm{Ar}$ & 34.041 & -4.32 & 65420 \\
\hline
\end{tabular}


Table 3: Prior distribution for the model parameters of the rate coefficients. The intervals for the uniform priors are chosen based on past studies [46]

\begin{tabular}{c|ccc}
\hline- & $p\left(A_{i} \mid M\right)$ & $p\left(m_{i} \mid M\right)$ & $p\left(\Theta_{i} \mid M\right)$ \\
\hline$R 1$ & $\mathrm{U}[14,21]$ & $\mathrm{U}[-3,1]$ & $\mathrm{U}[35000,70000]$ \\
$R 2$ & $\mathrm{U}[8,16]$ & $\mathrm{U}[-2,1]$ & $\mathrm{U}[5000,12000]$ \\
$R 3$ & $\mathrm{U}[12,14]$ & $\mathrm{U}[-2,1]$ & $\mathrm{U}[-1000,1000]$ \\
$R 4$ & $\mathrm{U}[11,14]$ & $\mathrm{U}[-1,2]$ & $\mathrm{U}[5000,15000]$ \\
$R 5$ & $\mathrm{U}[12,19]$ & $\mathrm{U}[-3,1]$ & $\mathrm{U}[10000,40000]$ \\
$R 6$ & $\mathrm{U}[7,14]$ & $\mathrm{U}[-1,3]$ & $\mathrm{U}[2000,6000]$ \\
\hline
\end{tabular}

Table 4: Posterior parameter correlation coefficient and partial correlation coefficient matrices for case 1 . The upper diagonal entries show the correlation coefficients and the lower diagonal entries show the partial correlation coefficients

\begin{tabular}{c|cccc}
\hline- & $A_{1}$ & $m_{1}$ & $\Theta_{1}$ & $\sigma_{\text {total }}^{2}$ \\
\hline$A_{1}$ & 1.0000 & -0.9978 & 0.8871 & 0.0037 \\
$m_{1}$ & -1.000 & 1.0000 & -0.8548 & -0.0049 \\
$\Theta_{1}$ & 0.9977 & 0.9971 & 1.0000 & -0.0052 \\
$\sigma_{\text {total }}^{2}$ & 0.0074 & 0.0072 & -0.0086 & 1.0000 \\
\hline
\end{tabular}

Table 5: Posterior parameter correlation coefficient and partial correlation coefficient matrices for case 2 . The upper diagonal entries show the correlation coefficients and the lower diagonal entries show the partial correlation coefficients

\begin{tabular}{c|ccccccc}
\hline- & $A_{1}$ & $A_{3}$ & $m_{1}$ & $m_{3}$ & $\Theta_{1}$ & $\Theta_{3}$ & $\sigma_{\text {total }}^{2}$ \\
\hline$A_{1}$ & 1.0000 & 0.0022 & -0.9952 & 0.0022 & 0.8056 & -0.0490 & 0.0738 \\
$A_{3}$ & -0.9124 & 1.0000 & 0.0008 & -0.9762 & 0.0313 & -0.0090 & -0.0679 \\
$m_{1}$ & -1.0000 & -0.9151 & 1.0000 & -0.0191 & -0.7439 & 0.0273 & -0.0767 \\
$m_{3}$ & -0.9106 & -0.9992 & -0.9134 & 1.0000 & -0.1076 & 0.1777 & 0.0779 \\
$\Theta_{1}$ & 0.9975 & 0.8890 & 0.9968 & 0.8868 & 1.0000 & -0.1809 & 0.0369 \\
$\Theta_{3}$ & 0.9000 & 0.9701 & 0.9021 & 0.9705 & -0.8814 & 1.0000 & 0.0627 \\
$\sigma_{\text {total }}^{2}$ & 0.0040 & -0.0071 & 0.0034 & -0.0046 & -0.0056 & 0.0179 & 1.0000 \\
\hline
\end{tabular}


Table 6: Posterior parameter correlation coefficient and partial correlation coefficient matrices for case 3 . The upper diagonal entries show the correlation coefficients and the lower diagonal entries show the partial correlation coefficients

\begin{tabular}{c|cccc}
\hline- & $A_{1}$ & $A_{3}$ & $A_{4}$ & $\sigma_{\text {total }}^{2}$ \\
\hline$A_{1}$ & 1.0000 & 0.0340 & 0.0373 & 0.0612 \\
$A_{3}$ & -0.9038 & 1.0000 & -0.0769 & 0.0115 \\
$A_{4}$ & -0.5498 & -0.3678 & 1.0000 & -0.0709 \\
$\sigma_{\text {total }}^{2}$ & -0.1100 & -0.1053 & 0.0865 & 1.0000 \\
\hline
\end{tabular}

Table 7: Posterior parameter correlation coefficient and partial correlation coefficient matrices for case 4 . The upper diagonal entries show the correlation coefficients and the lower diagonal entries show the partial correlation coefficients

\begin{tabular}{c|ccccccc}
\hline- & $A_{1}$ & $A_{2}$ & $A_{3}$ & $A_{4}$ & $A_{5}$ & $A_{6}$ & $\sigma_{\text {total }}^{2}$ \\
\hline$A_{1}$ & 1.0000 & -0.0256 & 0.0765 & -0.0109 & 0.0519 & 0.0589 & 0.1347 \\
$A_{2}$ & -0.8888 & 1.0000 & 0.0709 & 0.0257 & 0.0068 & -0.0176 & 0.0566 \\
$A_{3}$ & -0.8637 & -0.7107 & 1.0000 & 0.0864 & -0.0407 & 0.0269 & -0.0025 \\
$A_{4}$ & -0.5538 & -0.3234 & -0.3664 & 1.0000 & 0.0112 & 0.0541 & 0.0087 \\
$A_{5}$ & -0.1894 & -0.1420 & -0.1459 & -0.1581 & 1.0000 & -0.0481 & 0.0044 \\
$A_{6}$ & -0.1157 & -0.0935 & -0.1814 & -0.6861 & -0.0416 & 1.0000 & 0.0708 \\
$\sigma_{\text {total }}^{2}$ & -0.1417 & 0.1167 & 0.1285 & 0.0988 & 0.0348 & -0.1097 & 1.0000 \\
\hline
\end{tabular}




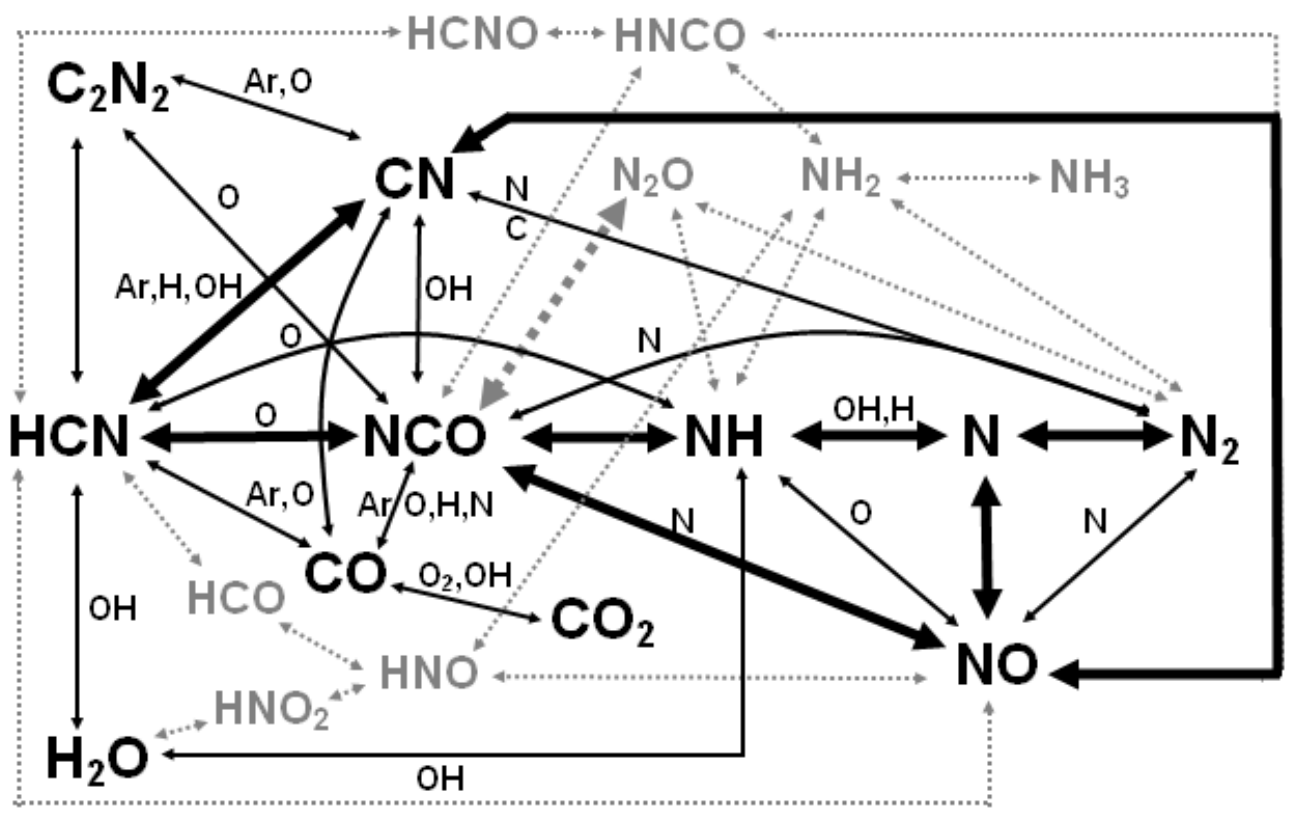

Figure 1: Reaction mechanism of $\mathrm{HCN} / \mathrm{O}_{2} / \mathrm{Ar}$ mixture proposed by Thielen and Roth (1987) (black line) [1] and some other possible important reactions proposed by other researchers (grey line) $[2,4,10]$

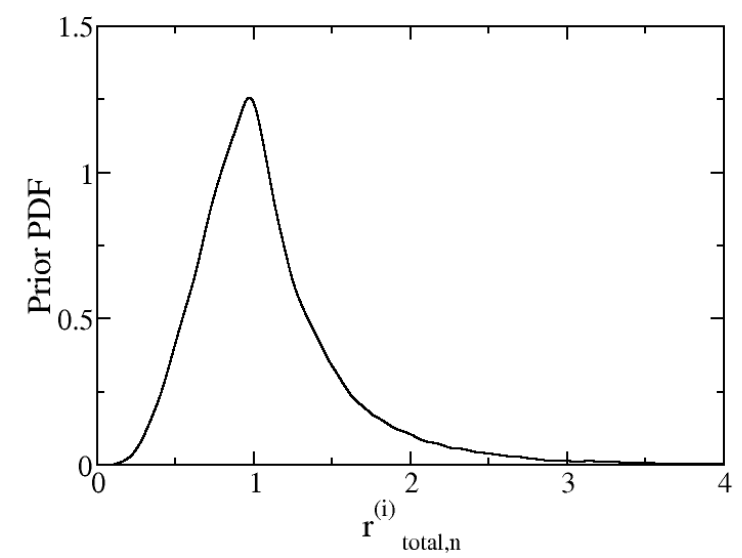

Figure 2: The prior PDF of the output total error $r_{\text {total }, n}^{(i)}$ at time $t_{n}$ for the $i$-th experiment 

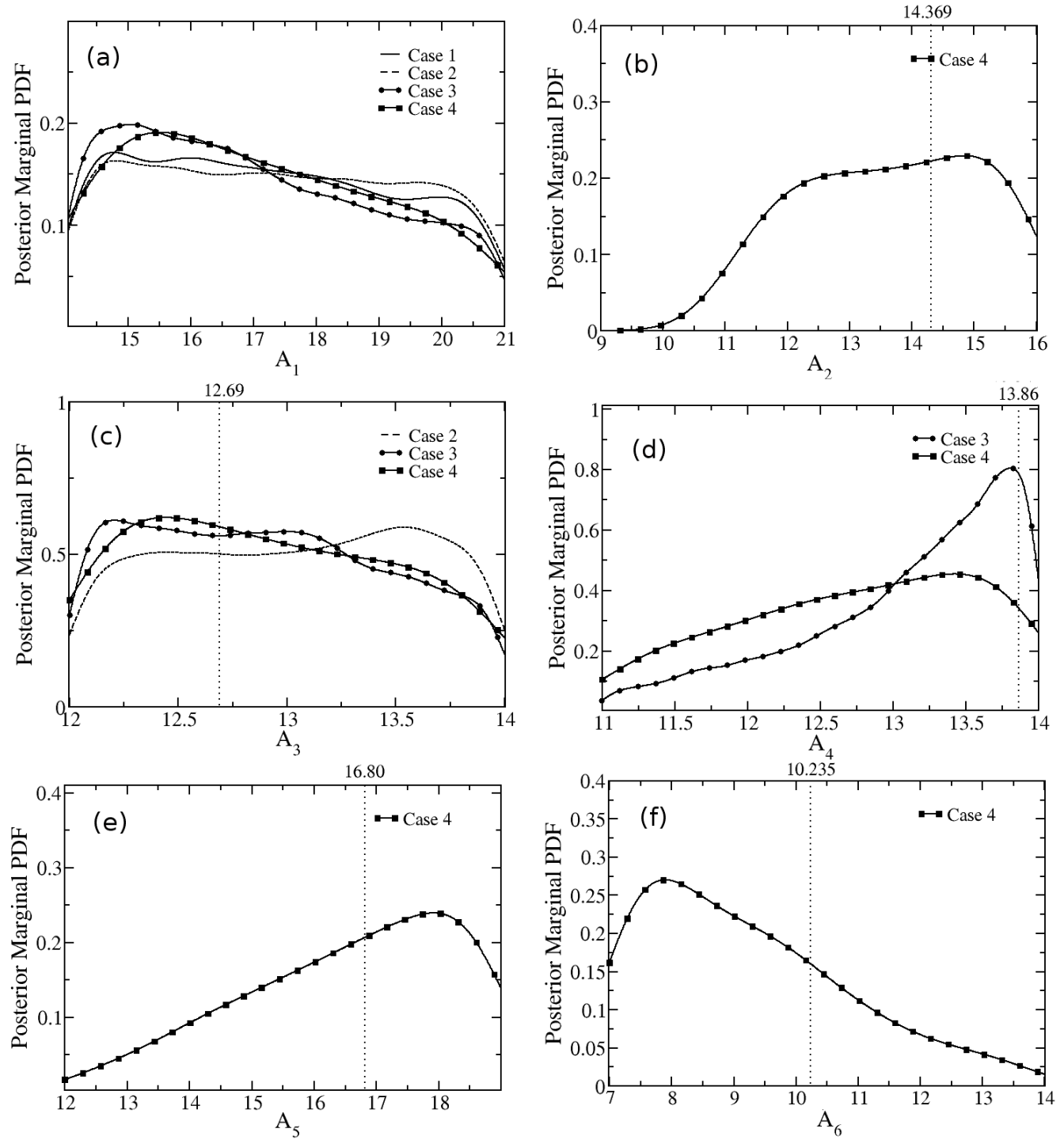

Figure 3: Posterior marginal PDFs for (a) $A_{1}$, (b) $A_{2}$, (c) $A_{3}$, (d) $A_{4}$, (e) $A_{5}$, and (f) $A_{6}$. Nominal values are shown on the top (dotted lines). 

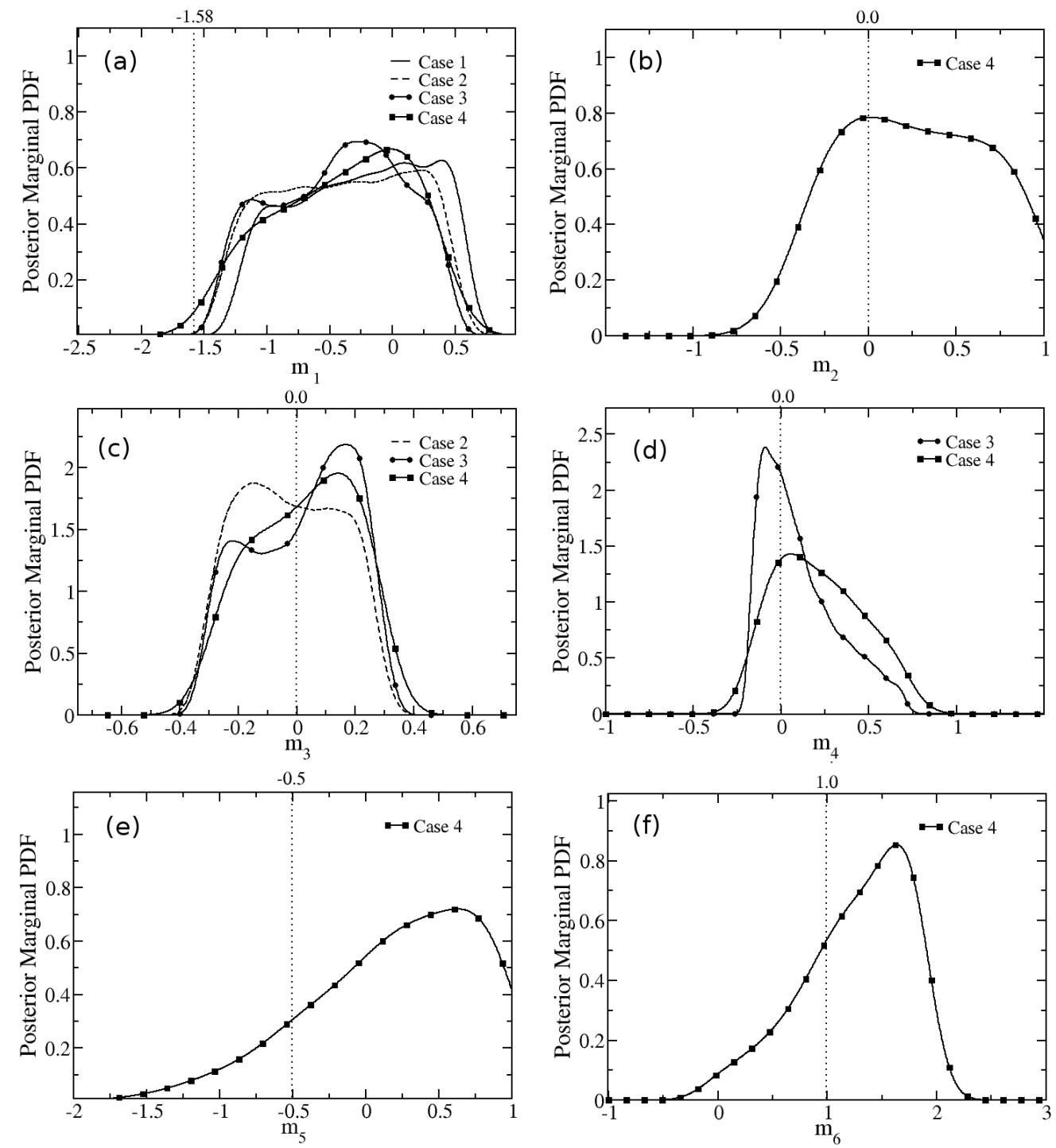

Figure 4: Posterior marginal PDFs for (a) $m_{1}$, (b) $m_{2}$, (c) $m_{3}$, (d) $m_{4}$, (e) $m_{5}$, and (f) $m_{6}$. Nominal values are shown on the top (dotted lines). 

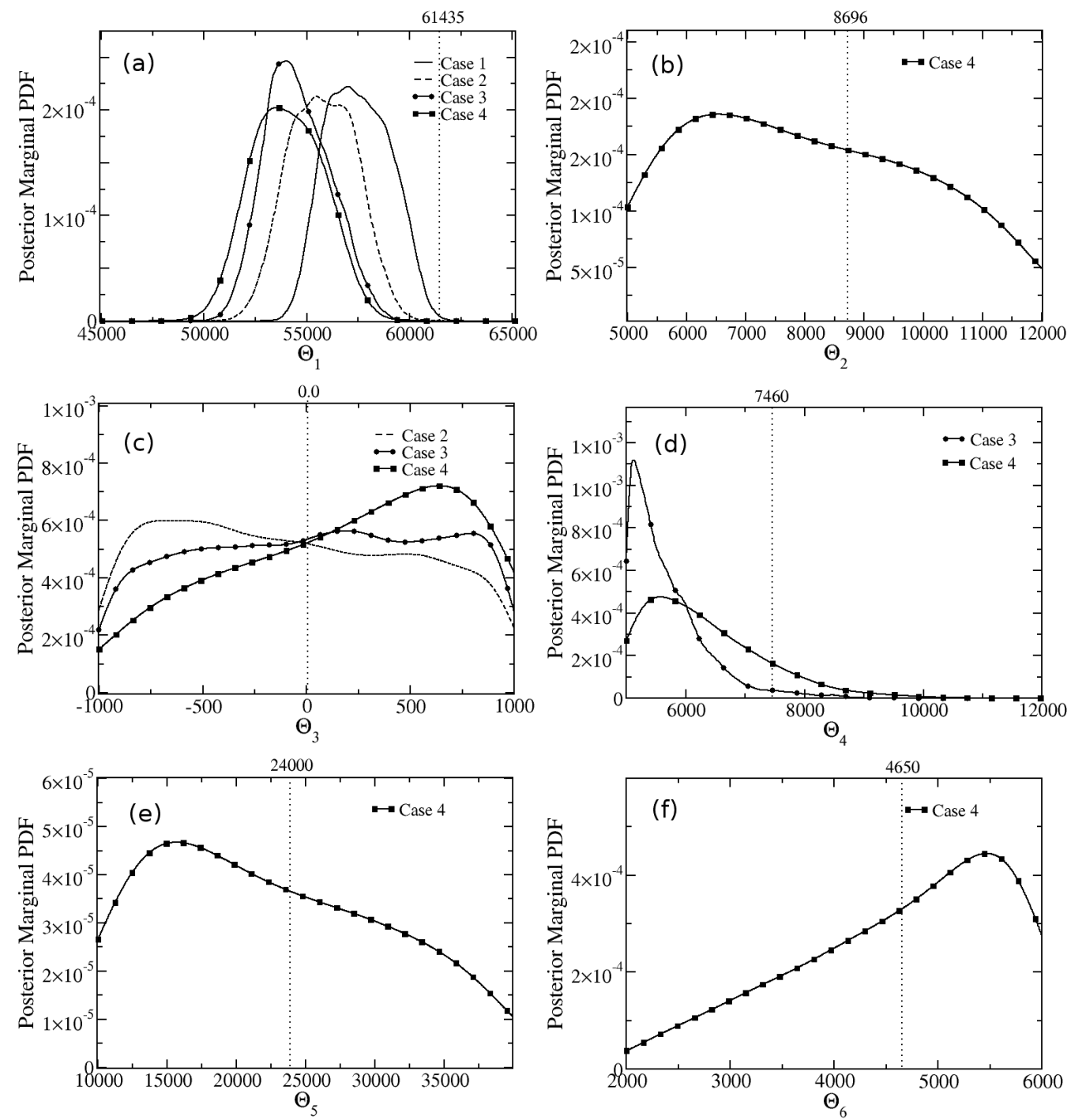

Figure 5: Posterior marginal PDFs for (a) $\Theta_{1}$, (b) $\Theta_{2}$, (c) $\Theta_{3}$, (d) $\Theta_{4}$, (e) $\Theta_{5}$, and (f) $\Theta_{6}$. Nominal values are shown on the top (dotted lines). 


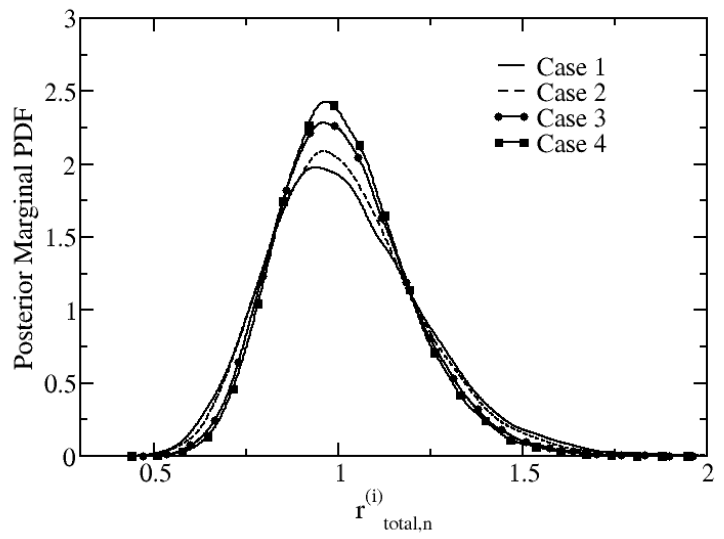

Figure 6: The posterior PDF of the prediction error $r_{\text {total, } n}^{(i)}$ at time $t_{n}$ subjected to the conditions of the $i$-th experiment 

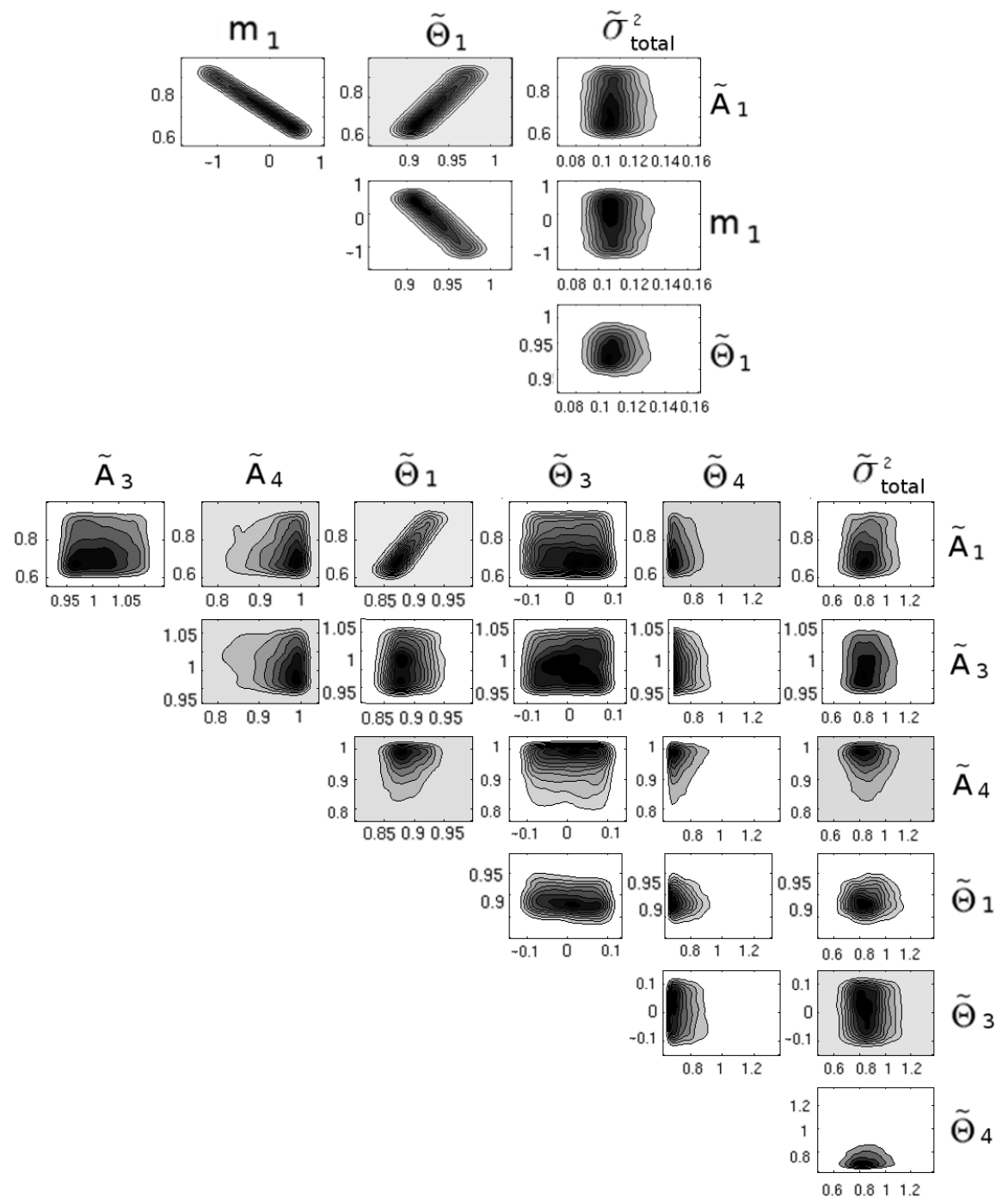

Figure 7: Posterior samples for some pairs of the uncertain parameters (a) case 1 and (b) case 3 

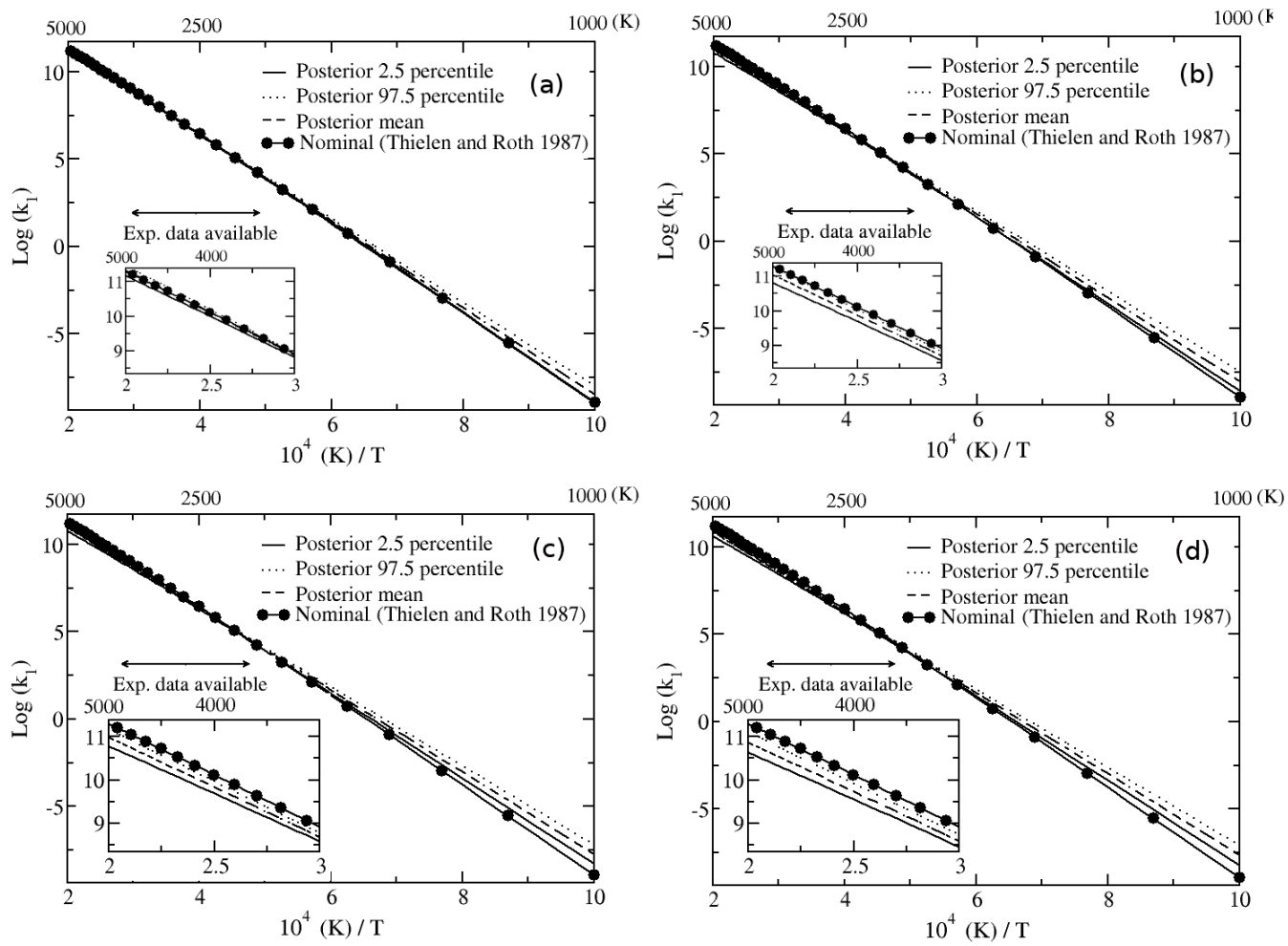

Figure 8: Posterior mean, 2.5 percentile and 97.5 percentile for the rate coefficient $\left(k_{1}\right)$ against $10^{4} \mathrm{~K} / T$, (a) case 1 , (b) case 2 , (c) case 3 , and (d) case 4 


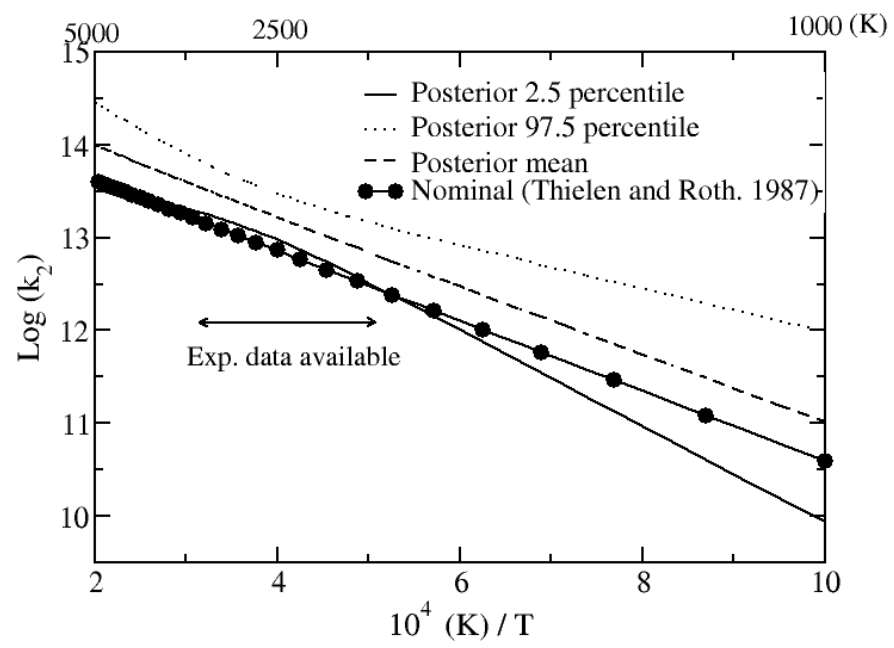

Figure 9: Posterior mean, 2.5 percentile and 97.5 percentile for the rate coefficient $\left(k_{2}\right)$ against $10^{4} \mathrm{~K} / T$ for case 4 

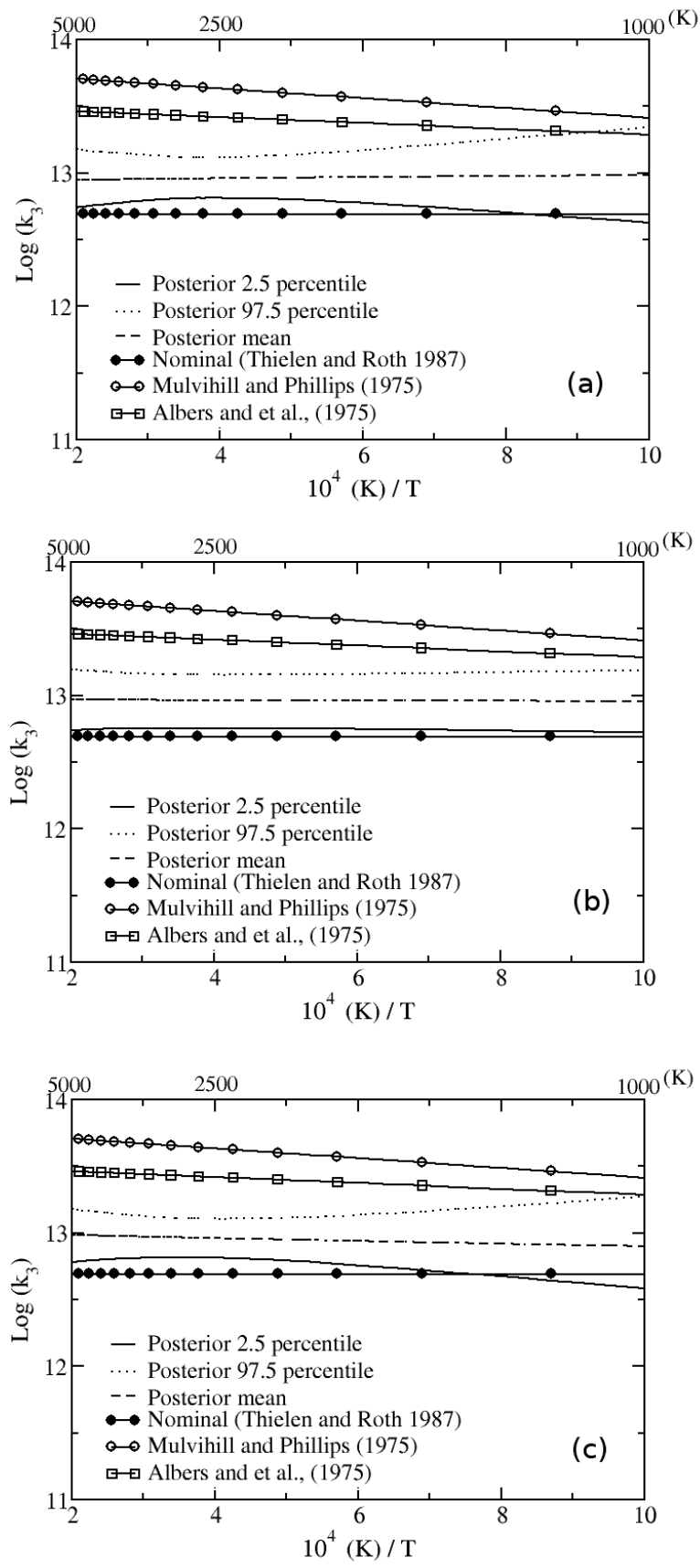

Figure 10: Posterior mean, 2.5 percentile and 97.5 percentile for the rate coefficient $\left(k_{3}\right)$ against $10^{4} \mathrm{~K} / T$ with experimental results $[8,55]$, (a) case 2 , (b) case 3 , and (c) case 4 

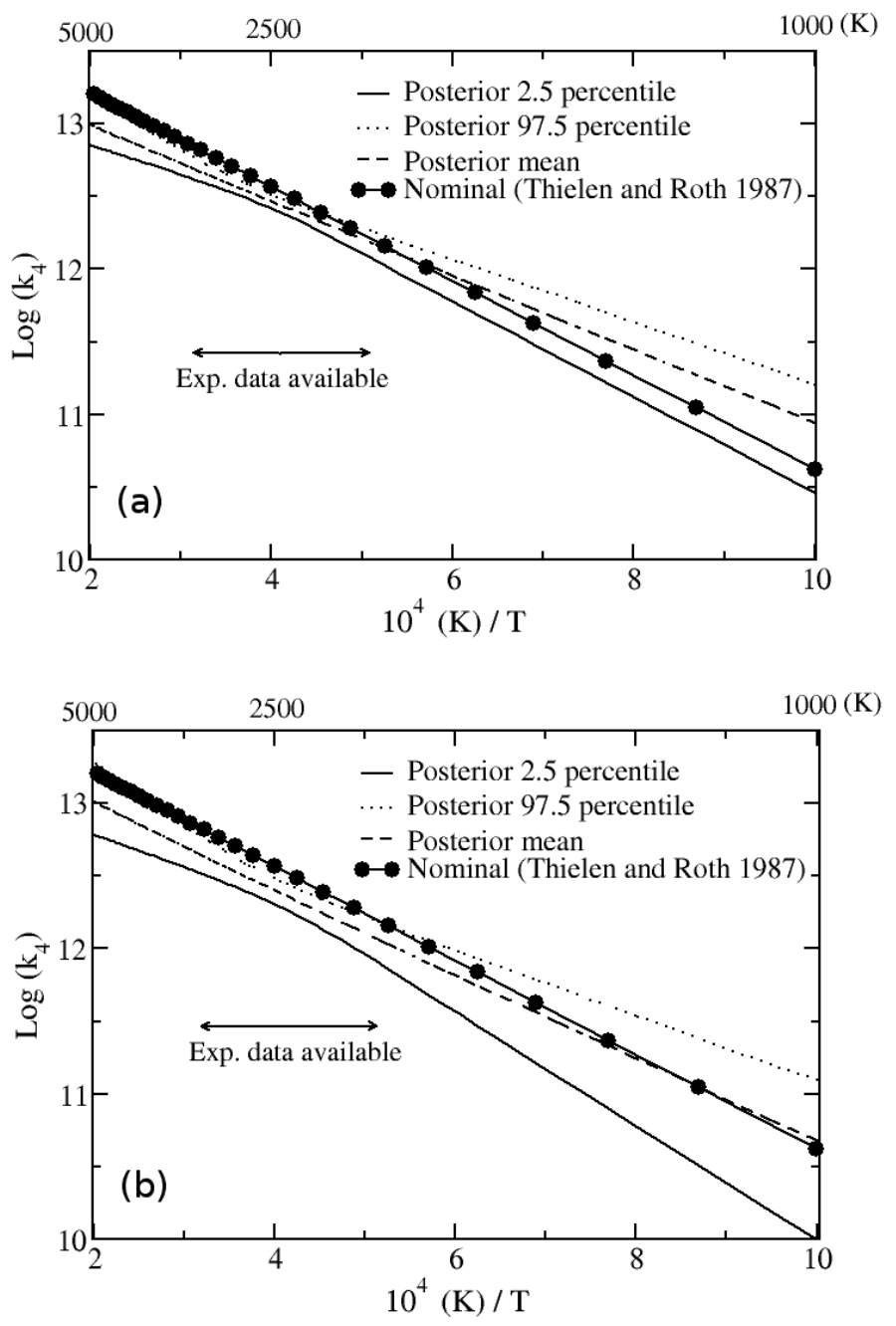

Figure 11: Posterior mean, 2.5 percentile and 97.5 percentile for the rate coefficient $\left(k_{4}\right)$ against $10^{4} \mathrm{~K} / T$, (a) case 3 and (b) case 4 


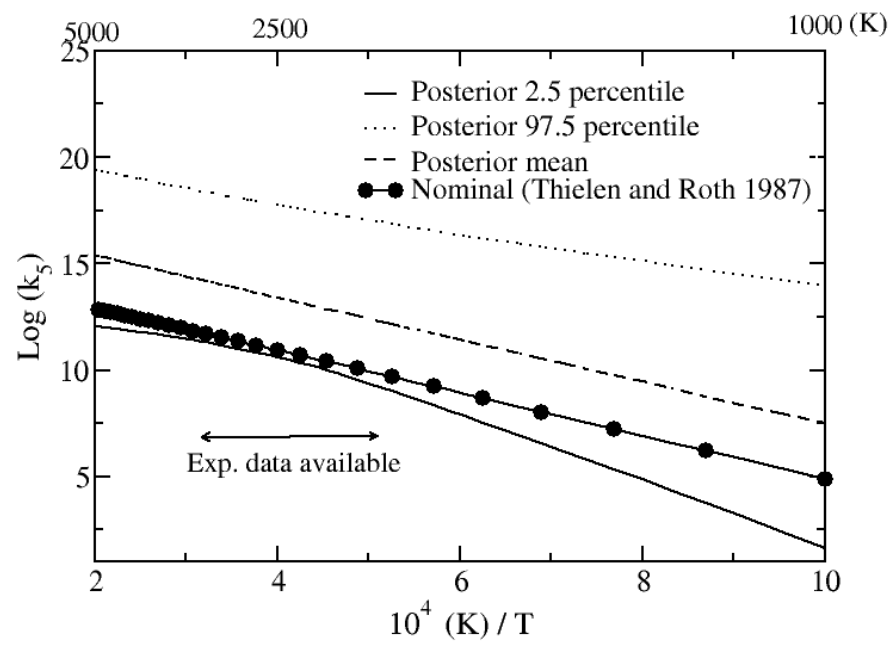

Figure 12: Posterior mean, 2.5 percentile and 97.5 percentile for the rate coefficient $\left(k_{5}\right)$ against $10^{4} \mathrm{~K} / T$ for case 4

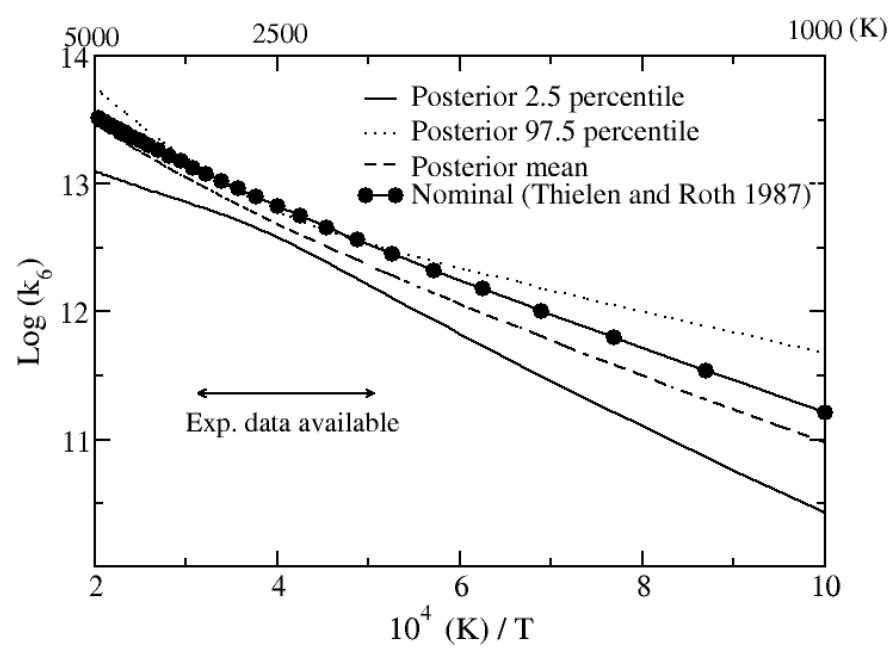

Figure 13: Posterior mean, 2.5 percentile and 97.5 percentile for the rate coefficient $\left(k_{6}\right)$ against $10^{4} \mathrm{~K} / T$ for case 4 


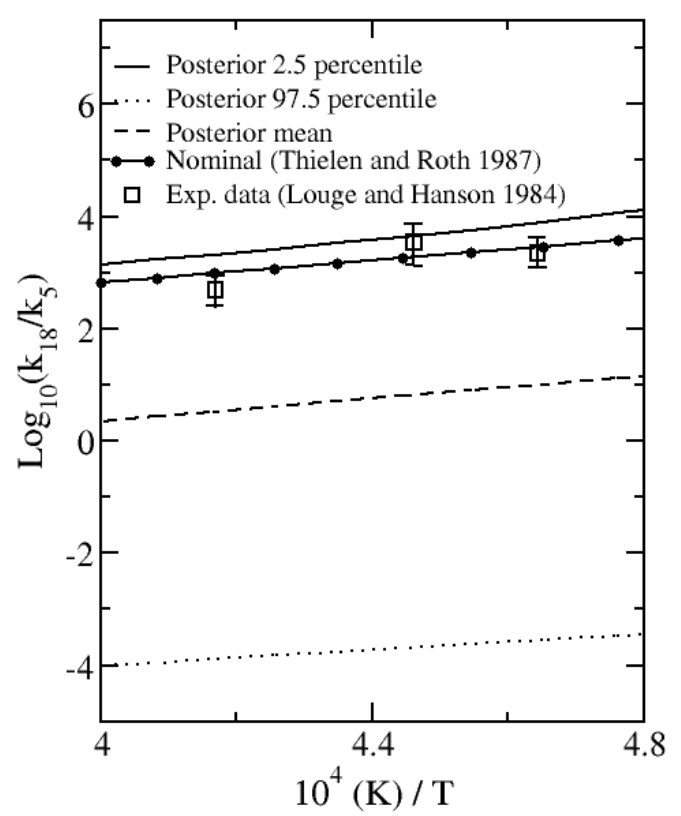

Figure 14: Posterior mean, 2.5 percentile and 97.5 percentile for $\left(k_{18} / k_{5}\right)$ against $10^{4} \mathrm{~K} / \mathrm{T}$ for case 4 

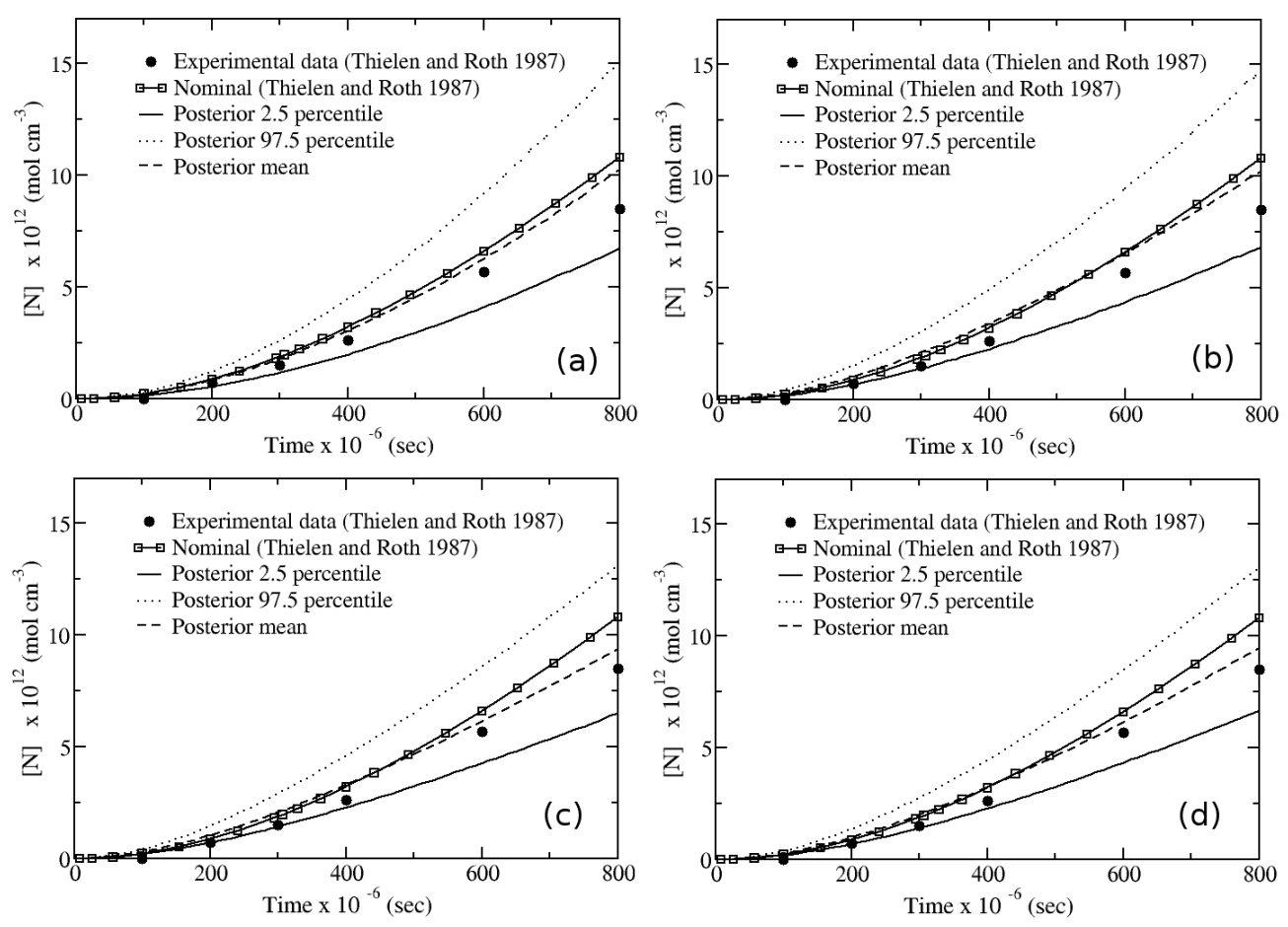

Figure 15: Posterior mean, 2.5 percentile and 97.5 percentile for the predicted concentration of nitrogen atom against time, (a) case 1, (b) case 2 ; (c) case 3 , and (d) case 4 . Initial conditions: the temperature is $2600 \mathrm{~K}$; the Argon concentration is $8.36 \mathrm{~mol} \mathrm{~cm}^{-3}$; the $\mathrm{HCN}$ concentration and the $\mathrm{O}_{2}$ concentration are $50 \mathrm{ppm}$ and $50 \mathrm{ppm}$ respectively. 

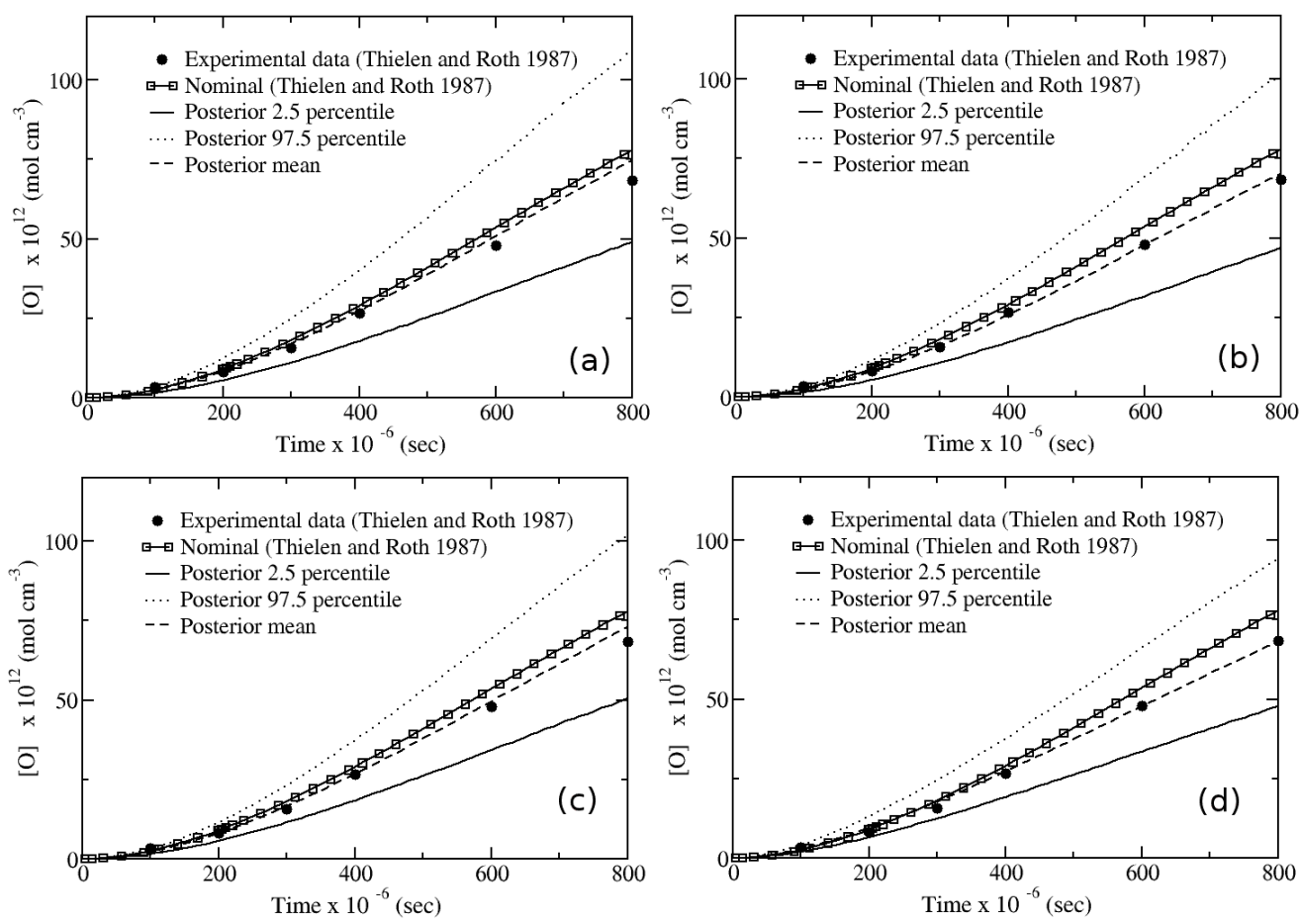

Figure 16: Posterior mean, 2.5 percentile and 97.5 percentile for the predicted concentration of oxygen atom against time, (a) case 1 , (b) case 2 ; (c) case 3 , and (d) case 4 . Initial conditions: the temperature is $2826 \mathrm{~K}$; the Argon concentration is $7.27 \mathrm{~mol} \mathrm{~cm}{ }^{-3}$; the $\mathrm{HCN}$ concentration and the $\mathrm{O}_{2}$ concentration are $50 \mathrm{ppm}$ and $50 \mathrm{ppm}$ respectively. 